\title{
ATR inhibition controls aggressive prostate tumors deficient in Y-linked histone demethylase KDM5D
}

\author{
Kazumasa Komura, ${ }^{1,2,3}$ Yuki Yoshikawa, ${ }^{1,2}$ Teppei Shimamura, ${ }^{4}$ Goutam Chakraborty, ${ }^{1}$ Travis A. Gerke, ${ }^{5}$ Kunihiko Hinohara, ${ }^{6}$ \\ Kalyani Chadalavada, ${ }^{7}$ Seong Ho Jeong, ${ }^{8}$ Joshua Armenia, ${ }^{9}$ Shin-Yi Du, ${ }^{1}$ Ying Z. Mazzu, ${ }^{1}$ Kohei Taniguchi, ${ }^{3,10}$ Naokazu Ibuki, ${ }^{2}$ \\ Clifford A. Meyer, ${ }^{11}$ Gouri J. Nanjangud, ${ }^{7}$ Teruo Inamoto, ${ }^{2}$ Gwo-Shu Mary Lee, ${ }^{6}$ Lorelei A. Mucci, ${ }^{12}$ Haruhito Azuma, ${ }^{2}$ \\ Christopher J. Sweeney, ${ }^{6}$ and Philip W. Kantoff'
}

\begin{abstract}
'Department of Medicine, Memorial Sloan Kettering Cancer Center, New York, New York, USA. ${ }^{2}$ Department of Urology and ${ }^{3}$ Translational Research Program, Osaka Medical College, Osaka, Japan. ${ }^{4}$ Division of Systems Biology, Nagoya University Graduate School of Medicine, Nagoya, Japan. ${ }^{5}$ Department of Cancer Epidemiology, Moffitt Cancer Center, Tampa, Florida, USA. ${ }^{6}$ Department of Medical Oncology, Dana-Farber Cancer Institute, Boston, Massachusetts, USA. ${ }^{7}$ Molecular Cytogenetics Core, Memorial Sloan Kettering Cancer Center, New York, New York, USA. ${ }^{8}$ Department of Medicine, Penn State College of Medicine, Hershey, Pennsylvania, USA. ${ }^{9} \mathrm{H} u m a n$ Oncology and Pathogenesis Program, Memorial Sloan Kettering Cancer Center, New York, New York, USA. ${ }^{10}$ Department of Ceneral and Gastroenterological Surgery, Osaka Medical College, Osaka, Japan. "Department of Biostatistics and Computational Biology, Dana-Farber Cancer Institute and Harvard T.H. Chan School of Public Health, Boston,
\end{abstract} Massachusetts, USA. ${ }^{2}$ Department of Epidemiology, Harvard T.H. Chan School of Public Health, Boston, Massachusetts, USA.

\begin{abstract}
Epigenetic modifications control cancer development and clonal evolution in various cancer types. Here, we show that loss of the male-specific histone demethylase lysine-specific demethylase 5D (KDM5D) encoded on the Y chromosome epigenetically modifies histone methylation marks and alters gene expression, resulting in aggressive prostate cancer. Fluorescent in situ hybridization demonstrated that segmental or total deletion of the $Y$ chromosome in prostate cancer cells is one of the causes of decreased KDM5D mRNA expression. The result of ChIP-sequencing analysis revealed that KDM5D preferably binds to promoter regions with coenrichment of the motifs of crucial transcription factors that regulate the cell cycle. Loss of KDM5D expression with dysregulated $\mathrm{H} 3 \mathrm{~K} 4 \mathrm{me}$ transcriptional marks was associated with acceleration of the cell cycle and mitotic entry, leading to increased DNA-replication stress. Analysis of multiple clinical data sets reproducibly showed that loss of expression of KDM5D confers a poorer prognosis. Notably, we also found stress-induced DNA damage on the serine/threonine protein kinase ATR with loss of KDM5D. In KDM5D-deficient cells, blocking ATR activity with an ATR inhibitor enhanced DNA damage, which led to subsequent apoptosis. These data start to elucidate the biological characteristics resulting from loss of KDM5D and also provide clues for a potential novel therapeutic approach for this subset of aggressive prostate cancer.
\end{abstract}

\section{Introduction}

Prostate cancer (PC) is the most common cancer among men in the United States and one of the leading causes of cancer death, with approximately 27,000 deaths per year from metastatic castrationresistant PC (mCRPC) (1). Elucidation and prevention of the biological mechanisms underlying the development of CRPC are urgently needed to guide development of better therapeutic strategies (2).

In prior work, we reported an association between decreased expression of the histone-modifying enzyme lysine-specific demethylase 5D (KDM5D) and resistance to docetaxel in PC in the presence of androgen (3). Moreover, the expression of KDM5D was found to be lower in CRPC tumors than in hormone-naive primary PC tumors, and CRPC patients with low tumor expression of KDM5D had shorter overall survival. KDM5D is a malespecific lysine-specific demethylase encoded on the Y chromosome that is capable of demethylating the active transcriptional marks H3K4me3 and H3K4me2 (4). Despite extensive genomewide analysis by next-generation sequencing (NGS), copy number

Conflict of interest: The authors have declared that no conflict of interest exists Submitted: August 8, 2017; Accepted: April 12, 2018.

Reference information: J Clin Invest. 2018;128(7):2979-2995.

https://doi.org/10.1172/JCI96769. alterations (CNA) of genes on the Y chromosome have not been well studied. Herein, we demonstrate that loss of KDM5D leads to an aggressive form of PC by epigenetic modifications that promote cell cycling and DNA-replication stress. Furthermore, we show the synthetic lethal approach of using a serine/threonine protein kinase ATR inhibitor in cells that have lost KDM5D.

\section{Results}

Copy number loss of KDM5D in PC. We observed reduced mRNA expression of KDM5D in CRPC compared with primary PC in a number of publicly available data sets (refs. 5-12 and Supplemental Figure 1; supplemental material available online with this article; https://doi.org/10.1172/JCI96769DS1). This might be due, at least in part, to the deletion of the KDM5D locus on the $\mathrm{Y}$ chromosome (3). This hypothesis was further supported by FISH findings in LNCaP, LNCaP-C42, and LNCaP-104R2 cells (LNCaP-derived CRPC cell lines) (Figure 1A and Supplemental Figure 2A). Compared with parental LNCaP cells, in which most cells $(87.5 \%)$ exhibit 2 copies of the Y chromosome, the Yp11/ Yq11 segment, which spans the KDM5D locus (Yq11), was uniformly lost in the androgen-independent LNCaP-104R2 cell line. Consistently, mRNA and protein expression of KDM5D in the LNCaP-104R2 cell line were absent (Figure 1B). Interestingly, 


\section{Table 1. Summary of FISH analysis in TMA}

\begin{tabular}{|c|c|c|}
\hline Variables & Number of cases & Positive for loss of KDM5D (\%) \\
\hline Normal prostate & 7 & 0 \\
\hline Adjacent normal prostate & 7 & 0 \\
\hline Adenocarcinoma & 75 & $8(11 \%)$ \\
\hline \multicolumn{3}{|l|}{ Tumor grading } \\
\hline $\mathrm{T} 1$ & 2 & 0 \\
\hline $\mathrm{T} 2$ & 37 & $5(14 \%)$ \\
\hline T3 & 20 & $1(5 \%)$ \\
\hline $\mathrm{T} 4$ & 3 & 0 \\
\hline Not known & 13 & $2(15 \%)$ \\
\hline \multicolumn{3}{|l|}{ Stage } \\
\hline 1 & 3 & 0 \\
\hline$\|$ & 32 & $5(16 \%)$ \\
\hline III & 14 & $1(8 \%)$ \\
\hline IV & 12 & 0 \\
\hline Not known & 14 & $2(13 \%)$ \\
\hline \multicolumn{3}{|l|}{ Gleason grade } \\
\hline 3 & 9 & 0 \\
\hline 4 & 30 & $1(3 \%)$ \\
\hline 5 & 29 & $7(24 \%)$ \\
\hline Not known & 7 & 0 \\
\hline
\end{tabular}

Patient information is provided in Supplemental Table 1.

a very small fraction of LNCaP cells $(0.5 \%)$ were devoid of the Yp11/Yq11 segment, and exclusion of the locus via micronuclei in LNCaP and LNCaP-C42 cells was suggestive of clonal selection of cells with loss of KDM5D (Figure 1C). In addition, CNA and gene expression profiling (GEP) data from the Grasso cohort (6) exhibited a positive correlation between CNA and mRNA expression levels of KDM5D (Supplemental Figure 2B), implying that loss of the KDM5D locus is likely the main cause of decreased KDM5D expression. FISH analysis on a commercially available formalinfixed paraffin-embedded (FFPE) tissue microarray (TMA) of primary human PC tissue revealed loss of KDM5D in 11\% of cases $(8 / 75)$, and the loss was restricted to higher Gleason grade cancers (Table 1, Supplemental Table 1). Of those 8 cases with loss, 2 cases showed an intact Yp locus, whereas 6 cases lost both the Yp and Yq segments (Figure 1D). Intratumor heterogeneity of the loss of KDM5D observed in 2 cases supported the hypothesis of clonal selection (Supplemental Figure 2C, Supplemental Table 2). We also performed RNA-sequencing (RNA-seq) of the Y chromosome genes in LNCaP and 22RV1 (Supplemental Figure 2D). Most of the $\mathrm{Y}$ chromosome genes were not transcribed. With the use of the Taylor cohort (9), we assessed the association between gene transcript levels close to the KDM5D gene on the Y chromosome (including RPS4Y1, UTY, and TTTY) in 131 primary tumors from PC patients on disease-free survival (DFS). In contrast to the findings of lower KDM5D levels correlating with shorter DFS, lower levels of RPS4Y1 (Yp11.2), UTY (Yq11.221), and TTTY (Yq11.221223) were not associated with a shorter DFS. These data support the cell-line analyses indicating deletion of the KDM5D locus on the Y chromosome might be a key feature of aggressive PC.

Aggressive phenotype in loss of KDM5D. The differential expression of KDM5D in LNCaP and LNCaP-104R2 enables the study of the biological function of KDM5D in PC, and RNA-seq analysis confirmed a different GEP in LNCaP compared with LNCaP104R2 cells (Supplemental Figure 3A). To explore the biological impact of loss of KDM5D in PC cells, we generated sh-KDM5D in LNCaP and overexpression of KDM5D in LNCaP-104R2 (Figure $2 \mathrm{~A}$ and Supplemental Figure 3B). Interestingly, knockdown of KDM5D in LNCaP cells showed enhanced cell growth in charcoal-stripped serum (CSS) media compared with LNCaP shcontrol cells, and overexpression of KDM5D in LNCaP-104R2 cells inhibited cell growth (Figure 2B). We also found that knockdown of KDM5D in LNCaP cells rendered the cells resistant to bicalutamide, a first-generation androgen receptor (AR) antagonist. Enzalutamide, a more potent AR antagonist, was resistant at lower doses, but there was no effect on abiraterone (an androgen synthesis inhibitor) efficacy (Supplemental Figure 3C). Soft agar colony formation assay exhibited anchorage-independent growth with knockdown of KDM5D in LNCaP (Figure 2, C and D), which further suggested a more aggressive phenotype associated with loss of KDM5D. We next determined whether reintroduction of KDM5D following KDM5D knockdown in LNCaP cells reverted the observed phenotype. As shown in Figure 2E, the shRNA construct targeting the $3^{\prime}$ UTR on KDM5D mRNA was integrated in LNCaP cells. A colony-formation assay consistently exhibited increased numbers of colonies with knockdown of KDM5D, whereas this phenotype was abrogated by the reintroduction of KDM5D (Figure 2F). To further explore these findings, we developed an in vivo orthotopic xenograft mouse model using a quantitative luminescence measurement system. After orthotopic inoculation, cells were allowed to form tumors for 2 weeks, followed by surgical castration, and then luciferase activity was measured every 2 weeks (Figure 2, G and H). The LNCaP cells with knockdown of KDM5D (sh-KDM5D for 3' UTR plus pLenti-GFP control) developed tumors in castrated mice (Figure 2I), and reintroduction of KDM5D (sh-KDM5D for 3' UTR plus p-Lenti-KDM5D) significantly inhibited tumor formation (Figure 2J). Taken together, these data suggest that decreased expression of KDM5D affects the efficacy of androgen-deprivation therapy (ADT) as well as cellular growth patterns, leading to a more aggressive phenotype.

Epigenetic modification by the loss of KDM5D. Since KDM5D has been reported to demethylate H3K4me3 and H3K4me2 (4, 13), we sought to elucidate the epigenetic changes associated with loss of KDM5D that rendered a more aggressive phenotype. There was a modest change in global $\mathrm{H} 3 \mathrm{~K} 4$ methylation protein levels with knockdown of KDM5D in LNCaP (Supplemental Figure 4A), suggesting that the epigenetic modification by the loss of KDM5D involves specific and local changes without rewriting global histone methylation patterns, as reported in the previous study of other KDM5 families (14). To further explore the function of KDM5D, ChIP-seq was performed. The result, using KDM5D antibody in LNCaP-sh-control cells, revealed that the genomic binding sites of KDM5D were mainly located in the promoter regions of genes (Figure 3A and Supplemental Table 3). We then compared the KDM5D-binding sites with H3K4 methylation marks and identified an $\mathrm{H} 3 \mathrm{~K} 4 \mathrm{me} 3$ signal, signifying an active transcriptional mark, substantially colocalized with the KDM5D-binding region (Figure 3B). Next, to assess whether decreased KDM5D expression levels affected $\mathrm{H} 3 \mathrm{~K} 4 \mathrm{me} 3$ levels in those specific regions of 
A

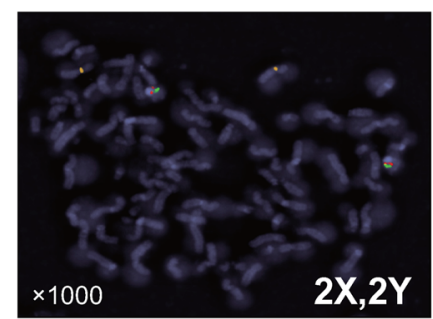

LNCaP-C42

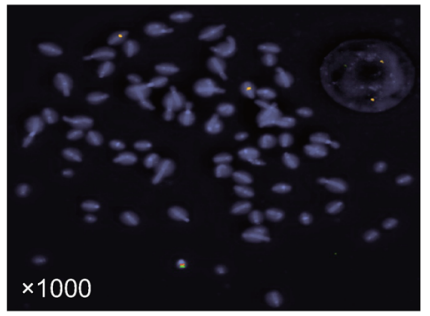

LNCaP-104R2

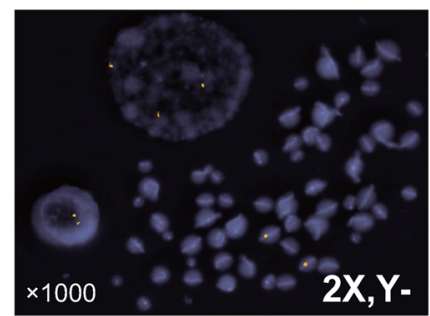

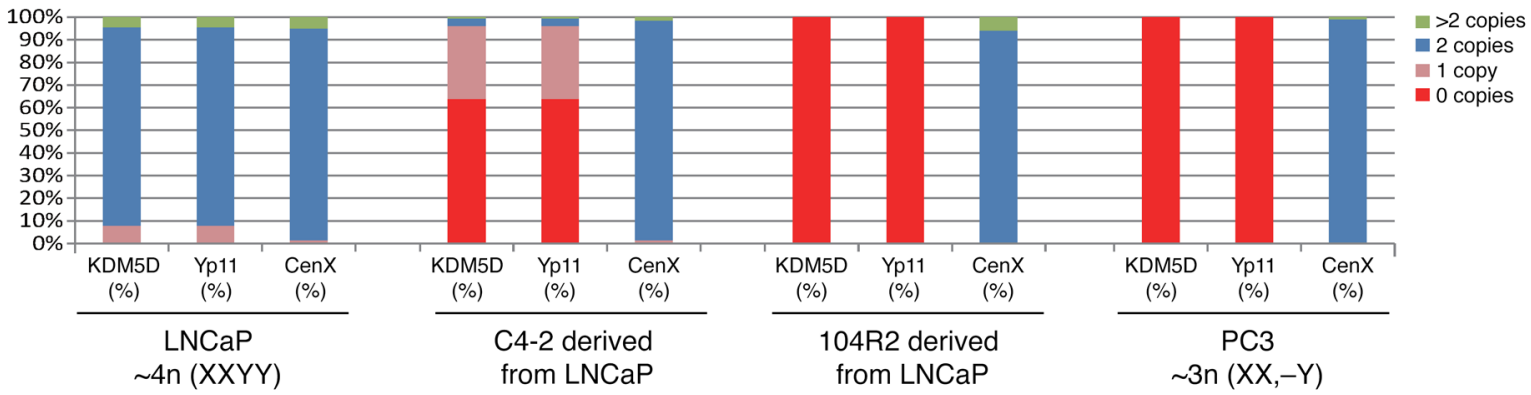
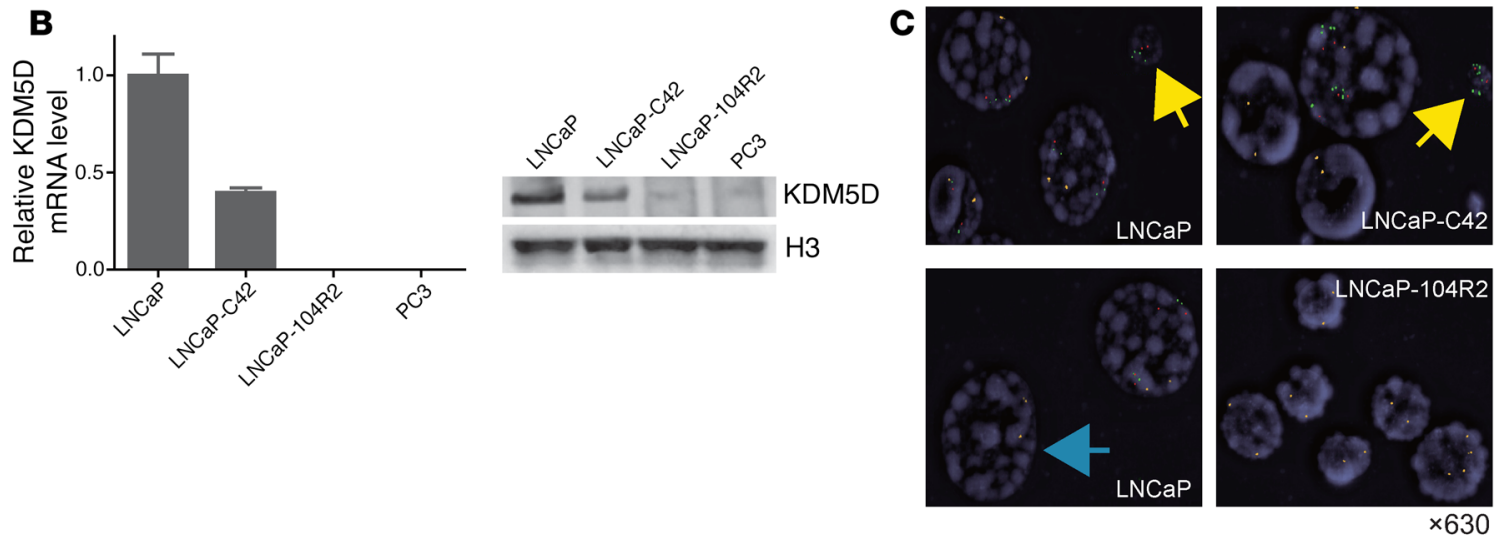

D

Prostate cancer (tissue ID: Mpr030207)

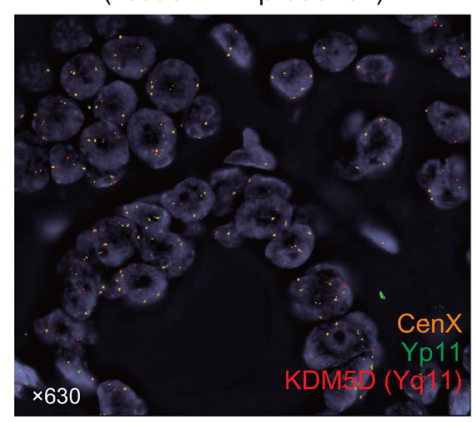

KDM5D +
Prostate cancer (tissue ID: Mpr060100)

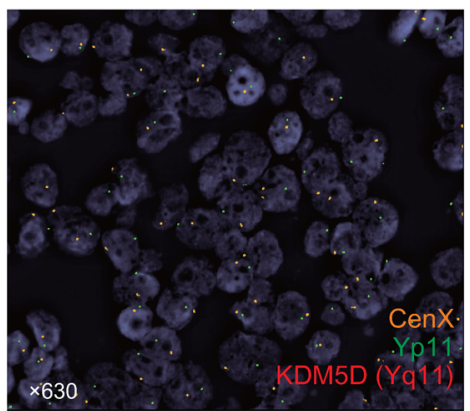

Loss of KDM5D(Yq11)
Prostate cancer (tissue ID: Mpr020323)

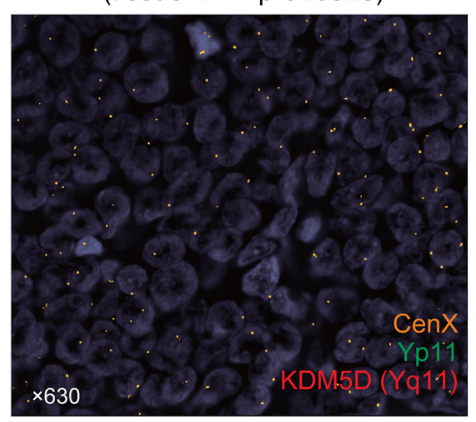

Loss of KDM5D(Yq11) / Yp11

Figure 1. Copy number loss of KDM5D in PC. (A) FISH analysis in LNCaP, LNCaP-C42, LNCaP-104R2, and PC3 cells using a 3-color KDM5D (Yq11: red)/ Yp11 (green)/CenX (orange) probe mix. PC3 cells that are known to have no KDM5D locus were used as negative control. (B) RNA expression of KDM5D in indicated cell lines. GAPDH was used for normalization. Mean expression values of 3 independent experiments were examined and are presented as mean \pm SD. For immunoblotting, nuclear fractions were collected in indicated cell lines and subjected to immunoblotting with the indicated antibodies. PC3, which is known to have no KDM5D locus, was used as a negative control. (C) FISH analysis using a 3-color KDM5D (Yq11: red)/Yp11 (green)/CenX (orange) probe mix. Yellow arrows indicate missegregation and/or exclusion of Yp11/ KDM5D (Yq11) via micronuclei (when some cells in metaphase translocate either the specific KDM5D locus or whole $Y$ chromosome to outside the nucleus, this DNA fragment is called micronuclei). Blue arrow indicates subpopulation with nullisomy of Y chromosome in LNCaP (0.5\%). (D) Representative image showing KDM5D-positive TMA and loss of KDM5D (Yq11) and KDM5D (Yq11)/Yp11 in TMA. 
A
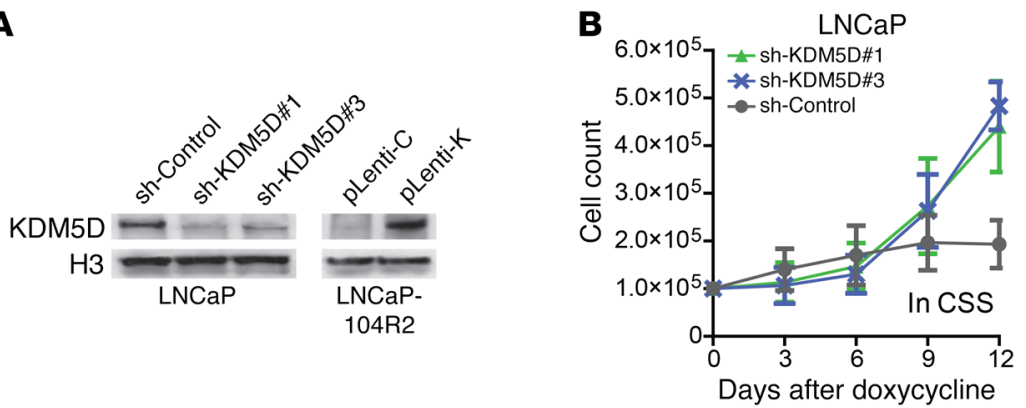

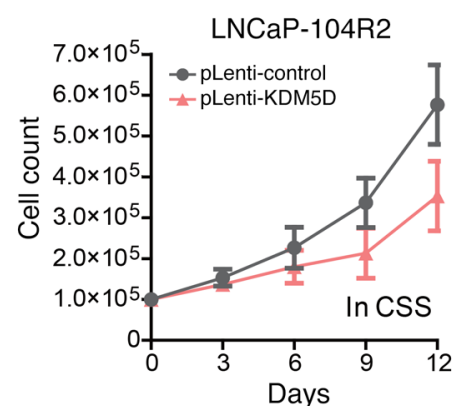

D
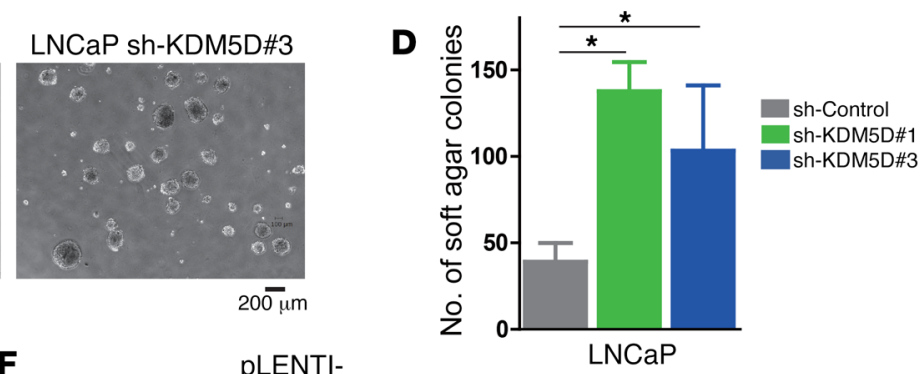

$20 \overline{\mathrm{um}}$

H
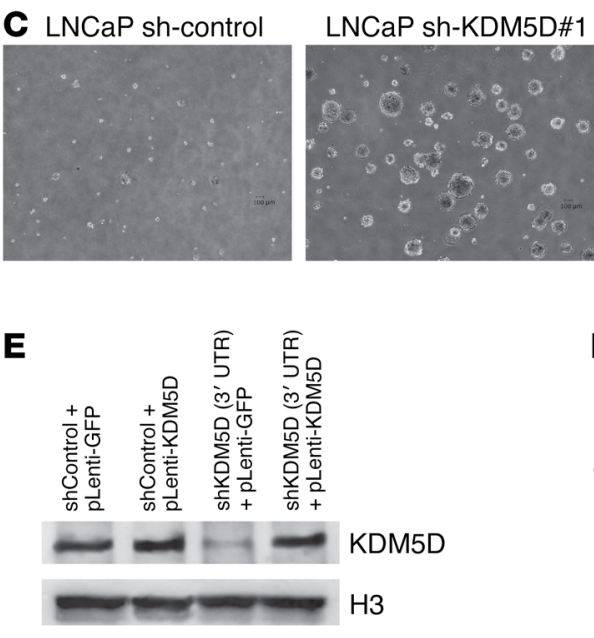

DM5D
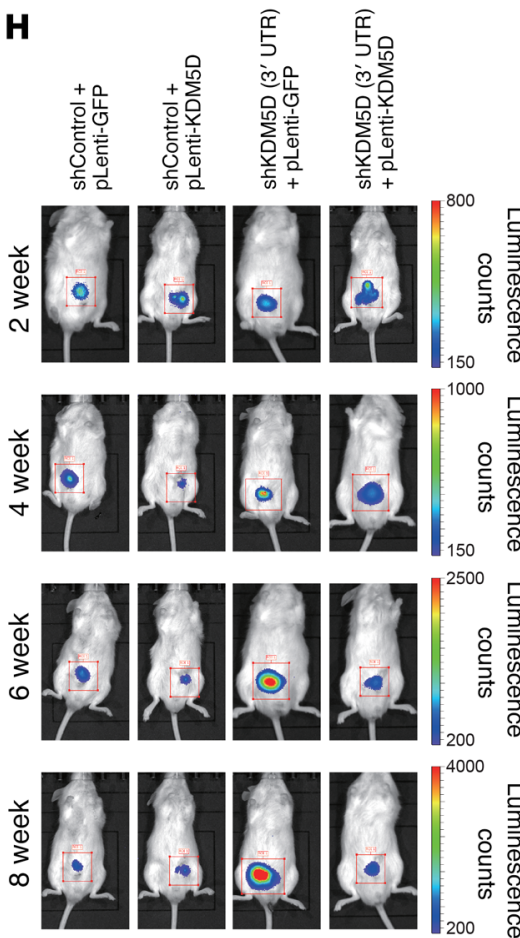

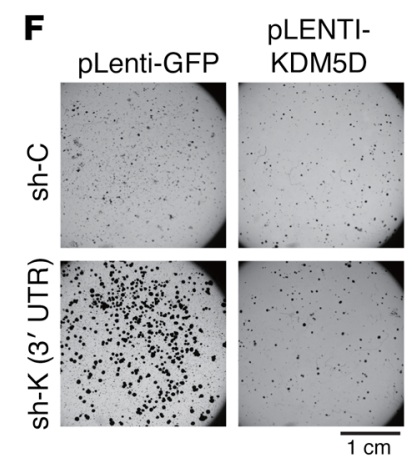

$$
\text { I }
$$
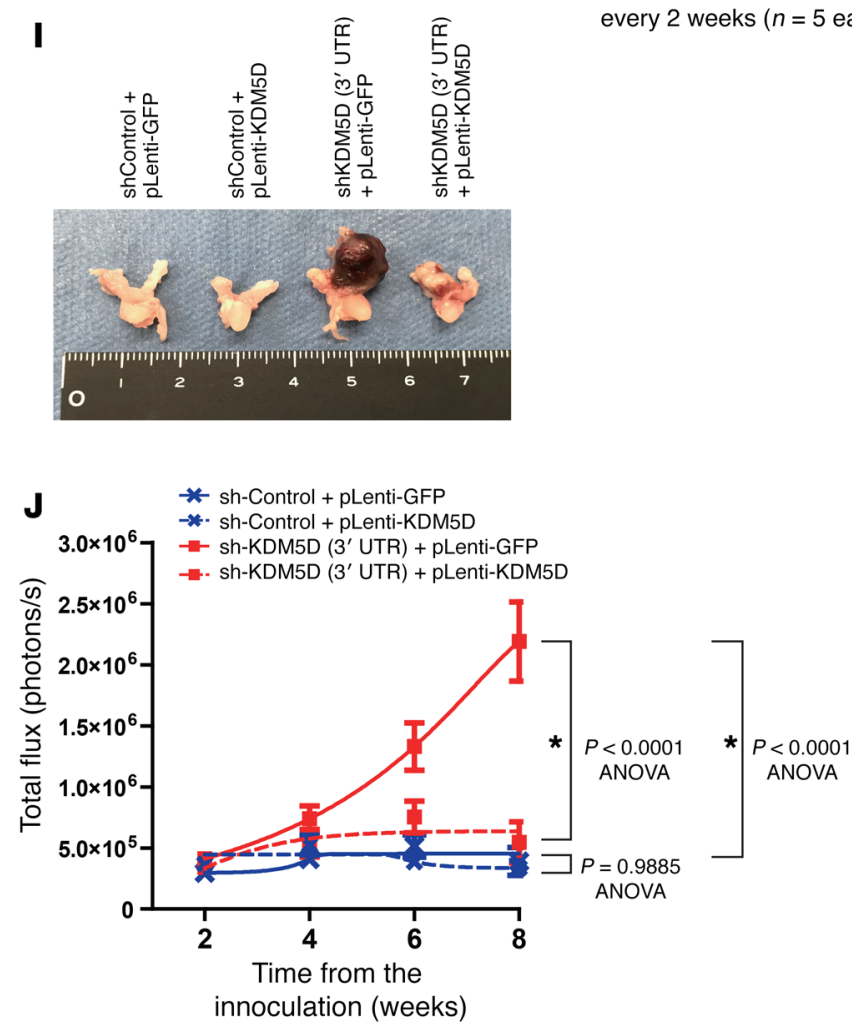
Figure 2. Aggressive phenotype in loss of KDM5D. (A) Immunoblotting in indicated cell lines. Nuclear fractions were collected in indicated cells and subjected to immunoblotting with the indicated antibodies. pLenti-C, control; pLenti-K, KDM5D. (B) Cell growth of indicated cell lines in CSS medium. LNCaP sh-control, KDM5D\#1, and KDM5D\#3 were cultured in 6-well plates with $0.15 \mu \mathrm{g} / \mathrm{ml}$ of doxycycline, and medium was changed every 3 days. 104R2-control and KDM5D overexpression cells were cultured in 6-well plates, and medium was changed every 3 days. (C) Soft agar colonyformation assay in LNCaP sh-control, KDM5D\#1, and KDM5D\#3. Representative images after 21 days are shown. (D) Number of colonies in C were counted in 5 random fields in 21 days, and results are shown as mean \pm SD. ${ }^{*} P<0.05$, 1-way ANOVA with post hoc Tukey's HSD test. (E) Immunoblotting in indicated cell lines. Nuclear fractions were collected in indicated cells and subjected to immunoblotting with the indicated antibodies. (F) Representative images of soft colony formation assay in indicated cell lines. (C) Schematic representation of orthotopic xenograft mouse model. After the orthotopic inoculation, cells were allowed to form the tumor in 2 weeks, followed by surgical castration, and then the luciferase activity was measured every 2 weeks ( $n=5$ in each group). (H) Representative images of the quantitative luminescence measurement for each group of 4 in an orthotopic xenograft model. (I) Representative images of the tumor orthotopically inoculated for 8 weeks in each group of 4 . (J) Quantitative evaluation of the developed tumor in orthotopic xenograft mice. Total flux (photons/s) in the region of interest (ROI) was recorded every 2 weeks. ${ }^{*} P<0.05,1$-way ANOVA with post hoc Tukey's HSD test.

the KDM5D-binding site, ChIP-seq in LNCaP sh-control and shKDM5D\#1 for H3K4me3, H3K4me2, and H3K4me1 were examined. Knockdown of KDM5D resulted in an increased $\mathrm{H} 3 \mathrm{~K} 4 \mathrm{me} 3$ signal in the KDM5D-binding region (Figure 3C). We also found increased $\mathrm{H} 3 \mathrm{~K} 4 \mathrm{me} 2$ and decreased $\mathrm{H} 3 \mathrm{~K} 4 \mathrm{me} 1$ signal with shKDM5D\#1 at these KDM5D-binding sites (Figure 3D), in line with previous reports showing that KDM5D is capable of demethylating H3K4me3 and H3K4me2, but not H3K4me1 $(4,13)$. To investigate the sequence specificity of KDM5D binding, we performed motif analysis of KDM5D-binding sites and found coenrichment of the motifs with crucial transcription factors for the cell cycle, such as E2F family and MYBL2 (Figure 3E). These data collectively indicated that KDM5D acts as an essential coregulator of multiple transcriptional factors by regulating the H3K4 methylation pattern in the promoter regions of genes.

Pathways affected by the modification of KDM5D expression levels. To further explore the mechanism by which loss of KDM5D contributes to an aggressive phenotype, we performed comprehensive transcriptome analysis by RNA-seq of LNCaP (control vs. sh-KDM5D\#1 and sh-KDM5D\#3) and LNCaP-104R2 (control vs. KDM5D overexpression), respectively. Since the KDM5 family members (A, B, C, and D) are known to have substantial overlap in their amino acid sequences (13), we first tested to determine whether modulation of the KDM5D expression level affected the expression of the other KDM5 family members. As shown in Supplemental Figure 4, B and C, no significant difference in expression was seen in other KDM5 family members when KDM5D expression level was altered. We then explored specific gene sets regulated by KDM5D, which may be the drivers of the more aggressive phenotype. Differentially expressed genes sorted by an FDR-adjusted $P$ value of less than 0.01 were compared between LNCaP cells in which KDM5D had been knocked down (sh-KDM5D\#1 and sh-KDM5D\#3) and in which KDM5D had been overexpressed (LnCaP-104R2) (Figure 4A). We found
143 overlapping genes, which were upregulated by knockdown of KDM5D in LNCaP and downregulated by overexpression of KDM5D in LNCaP-104R2, and 28 genes, which were downregulated by KDM5D knockdown in LNCaP and upregulated by overexpression of KDM5D in LNCaP-104R2 (Supplemental Tables 4 and 5). Notably, in gene ontology (GO), 143 genes negatively correlated with KDM5D expression levels were associated with 103 GO terms with an FDR of less than 0.04; cell-cycle-related terms were listed in the top 20 terms (Figure 4B and Supplemental Table 6), which was consistent with the result from the motif analysis in ChIP-seq. Next, Gene Set Enrichment Analysis (GSEA; (software. broadinstitute.org/gsea/index.jsp) (15) was performed to characterize the specific pathways affected by the transcriptome change with the KDM5D modification. We found 64 positively enriched pathways and 6 negatively enriched pathways with an FDR of less than 0.25 when KDM5D was knocked down in LNCaP (Supplemental Tables 7 and 8), whereas no positively enriched and 101 negatively enriched pathways at the same threshold were observed with overexpression of KDM5D in LNCaP-104R2 (Supplemental Table 9). Reassuringly, there was substantial overlap in the top 10 lists between the pathways upregulated with knockdown of KDM5D in LNCaP and those downregulated with overexpression of KDM5D in LNCaP-104R2 (Supplemental Figure 5), including DNA replication and mitotic-related pathways (the top 2 pathways) (Figure 4C). On the other hand, none of the pathways were duplicated between top 10 enrichment gene sets downregulated by knockdown of KDM5D in LNCaP and those upregulated by overexpression of KDM5D in LNCaP-104R2 cells. We then orthogonally validated the mRNA and ChIP quantitative PCR (qPCR) in MCM10 (listed in DNA-replication pathway) and NUF2 (listed in Mitotic_M_M_G1_Phases pathway) and confirmed that the loss of KDM5D altered $\mathrm{H} 3 \mathrm{~K} 4 \mathrm{me} 3$ levels in the promoter regions of genes, leading to aberrant mRNA expression levels (Figure 4D). Overexpression of KDM5D in LNCaP-104R2 cells inversely affected H3K4me3 signals, resulting in decreased mRNA expression levels in both MCM10 and NUF2. Taken together, these data indicate that loss of KDM5D epigenetically alters active transcriptional $\mathrm{H} 3 \mathrm{~K} 4 \mathrm{me} 3$ marks in the promoter regions for multiple transcriptional factors related to cell cycling, leading to the disruption of tightly coordinated DNA replication and mitotic programs.

Aberrant DNA damage during $S$ phase leading to DNA-replication stress. DNA replication and mitotic activity are tightly coordinated, and dysregulation of these processes has been reported as DNA-replication stress in various types of cancer $(16,17)$. There has also been accumulating evidence that DNA-replication stress causes continuous DNA damage and is associated with a worse clinical outcome $(18,19)$. To test the hypothesis that loss of KDM5D causes DNA-replication stress as well as continuous DNA damage, we assessed several replication stress and DNA damage markers. We found increased $\mathrm{rH} 2 \mathrm{AX}$ (a DNA-damage marker) and phosphorylated replication protein A2 (p-RPA2) (chromatin-bound ssDNA-binding protein) $(20,21)$ levels in KDM5D KD in LNCaP cells (Figure 5A). We also found an accumulated $\mathrm{S}$ phase population with KDM5D knockdown (Figure $5 \mathrm{~B})$, suggesting that the replication stress by the loss of KDM5D slows the $\mathrm{S}$ phase to manage DNA damage with increasing stalled replication forks, as shown by $\mathrm{rH} 2 \mathrm{AX}$ and $\mathrm{p}-\mathrm{RPA} 2$ protein levels. 
A

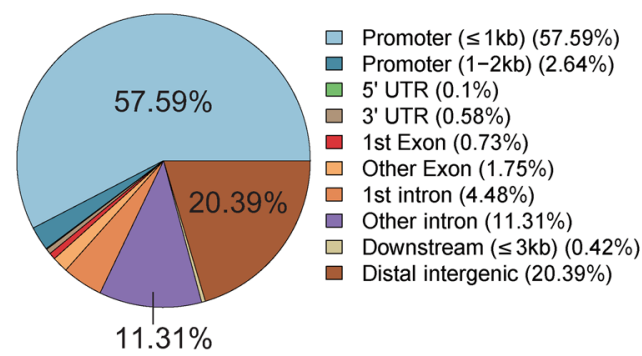

B

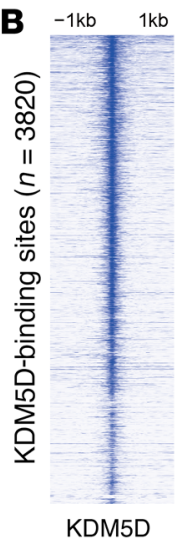

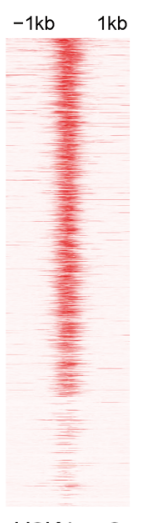

H3K4me3 H3K4me2 H3K4me1
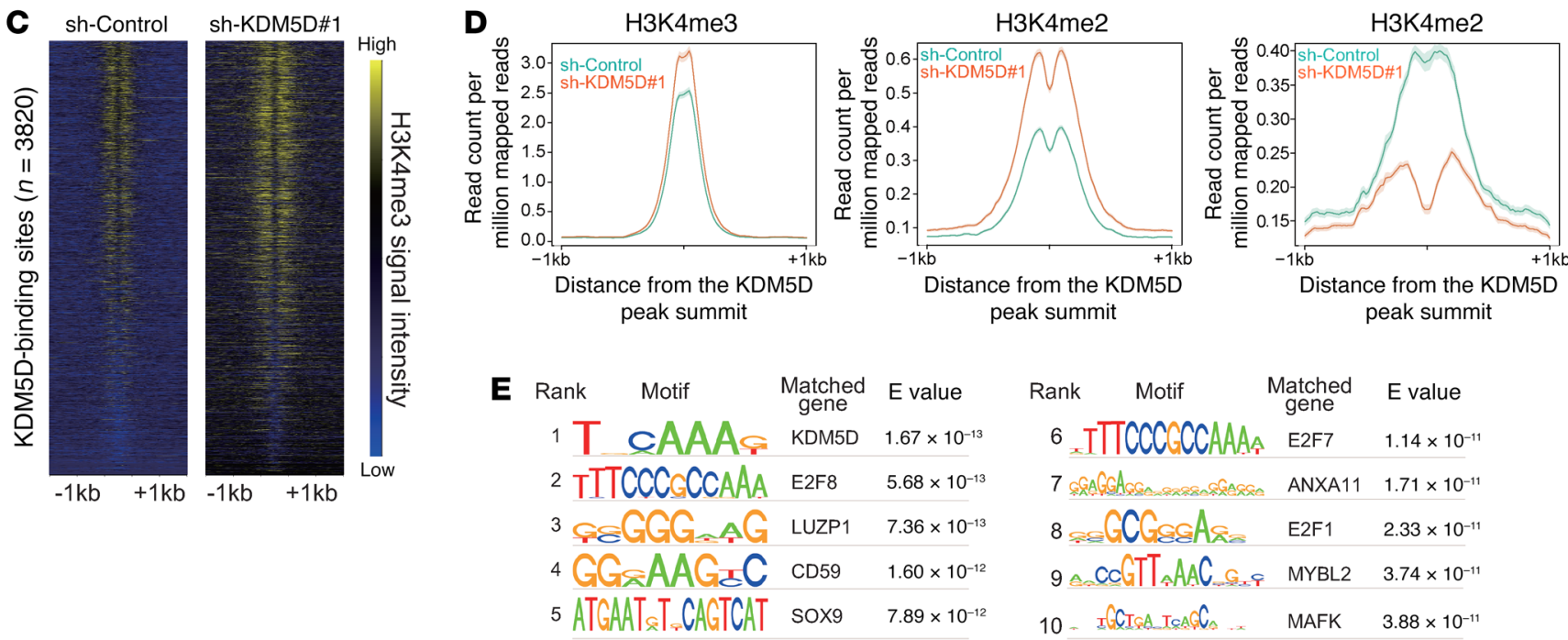

Figure 3. Epigenetic modification by the loss of KDM5D. (A) Pie chart of KDM5D-binding peak location on the genome $(n=3820)$. (B) Heatmap of KDM5D, H3K4me3, H3K4me2, and H3K4me1 around KDM5D-binding peak summit (-1 kb to $1 \mathrm{~kb}$ ) in LNCaP sh-control cells. (C) Heatmap of H3K4me3 signals around KDM5D-binding peak summit (-1 kb to $1 \mathrm{~kb})$ in LNCaP sh-control and sh-KDM5D\#1 cells. (D) Signal profile of H3K4me3, H3K4me2, and H3K4me1 around KDM5D peak summit in LNCaP sh-control and sh-KDM5D\#1 cells. (E) Top 10 enriched motifs on KDM5D-binding sites in LNCaP sh-control cells. KDM5D was validated as a top enriched gene. Motifs are listed according to expectation value (E value).

Indeed, as reported in the previous studies describing upregulation of DNA-repair pathways following aberrant DNA-replication activity $(22,23)$, DNA-repair pathways were also positively enriched with KDM5D knockdown in LNCaP and negatively enriched with overexpression of KDM5D in 104R2 cells (Figure 5C). Cisplatin, an agent that induces DNA double-strand breaks, was less effective with KDM5D knockdown in LNCaP, and overexpression of KDM5D sensitized 104R2 cells to cisplatin.

Evasion from $G_{2} / M$ arrest with ATR activation in cells with loss of KDM5D. Activated ATR signaling has been shown to have a central role in preventing the collapse of stalled replication forks due to DNA-replication stress (24), which in turn prevents massive double-strand breaks leading to cell death $(20,25)$. In addition, the ATR/CHK1/CDC25C/cyclin B1/CDK1 pathway is a well-studied cell-cycle checkpoint $(26,27)$. Phosphorylated CHK1 caused by ATR activation inactivates CDC25C. This leads to inhibition of CDK1 activity, which results in the prevention of mitotic entry, specifically as the result of $\mathrm{G}_{2} / \mathrm{M}$ arrest by activated ATR. To address how the cells that lost KDM5D simultaneously evade cell-cycle arrest in response to replication stress, we further analyzed the data from our cell lines. In our data, 143 genes were identified as genes negatively correlated with KDM5D expression (Figure 4A). We compared 138 genes listed in the $\mathrm{G}_{2} / \mathrm{M}$ checkpoint (MSigDB systematic name: M14052) with those 143 genes, and 7 genes were identified as upregulated genes by decreased KDM5D expression levels for the $\mathrm{G}_{2} / \mathrm{M}$ checkpoint, including CDK1 and CDC25C (Figure 6A). Immunoblotting showed increased p-ATR as well as elevated CDK1 and CDC25C protein levels in cells with knockdown of KDM5D (Figure 6, B and C). The DNA-damage markers rH2AX and p-histone $\mathrm{H} 3$, a marker of proliferation phosphorylated during mitosis (28), were colocalized with knockdown of KDM5D (Supplemental Figure 6A), suggesting their cooperation in replication stress-induced DNA damage and ongoing mitotic entry. We further reviewed our ChIP-seq data and validated increased H3K4me3 levels in the promoter regions of CDK1 and CDC25C with the loss of KDM5D and confirmed that KDM5D binds to those promoter regions mediating the chromatin status, including H3K4me3 (Figure 6, D and E). In the Taylor cohort, there was a sig- 
nificant negative correlation of mRNA expression levels between KDM5D and those mediators (Figure 6F). We also found a trend in several databases $(6,9,29)$ showing that lower expression of KDM5D is associated with more CNAs, suggesting its contribution to genomic instability (Supplemental Figure 6B), which has been reported as a consequence of DNA-replication stress (30, 31). Collectively, these data indicate that the loss of KDM5D causes subsequent DNA-replication stress, resulting in activated ATR signaling as well as concomitant activation of $\mathrm{G}_{2} / \mathrm{M}$ checkpoint mediators by altering histone methylation profiles in those promoter regions.

Synthetic lethal approach exploiting DNA-replication stress by loss of KDM5D. Activated ATR signaling precludes the collapse of stalled replication forks under replication stress. Given the fact that knockdown of KDM5D led to ATR activation, we next tested VE822, which is the first selective ATR inhibitor to enter clinical development (ClinicalTrials.gov, NCT02157792 in phase 1 and NCT02567409 in phase 2) and is now known as VX-970 (32), to assess its efficacy in KDM5D-knockdown PC cells. Compared with LNCaP control cells, treatment with VE822 in KDM5D KD decreased cell proliferation (Figure 7A) and enhanced DNA damage shown by increased $\mathrm{rH} 2 \mathrm{AX}$ expression as well as increased cleaved PARP expression, indicating increased apoptosis (Figure $7 \mathrm{~B})$. To validate the result in an additional model, VCaP cells, which express KDM5D, were employed and the effects of a KDM5D-targeting siRNA were evaluated. Increased p-ATR and p-CHK1, downstream targets of ATR, were confirmed in cells treated with KDM5D-targeting siRNA (siKDM5D cells) (Figure 7C). The accumulation of $\mathrm{rH} 2 \mathrm{AX}$ and $\mathrm{p}-\mathrm{RPA} 2$ in the nucleus was consistently detected with knockdown of KDM5D in VCaP cells (Figure 7D), which was similar to the result in LNCaP cells (Figure $5 \mathrm{~A})$. Importantly, knockdown of KDM5D in VCaP cells also sensitized the cells to VE822 (Figure 7, E and F), a result that is consistent with the higher sensitivity to ATR inhbition observed in PC in response to loss of KDM5D. These data suggest that activated ATR signaling with the loss of KDM5D can be exploited to elicit synthetic lethality by ATR inhibition in PC cells.

Higher sensitivity of ATR inhibitor to PC cells deficient in KDM5D in an in vivo xenograft mouse model. We next examined the efficacy of VE822 treatment in a panel of PC cell lines with KDM5D status (Figure 8, A and B). The CNA status of KDM5D in those PC cell lines was shown in our previous study (3). PC cell lines with deletion of KDM5D showed greater sensitivity to VE822 treatment than those expressing KDM5D (Figure 8B). To further validate these results, we next examined the efficacy of VE822 in an in vivo xenograft model using KDM5D-expressing PC cell lines (LNCaP and 22RV1 cells) and KDM5D-deficient PC cells (LNCaP104R2 and E006AA cells). After the subcutaneous inoculation of these cells, tumors developed to a volume of more than $150 \mathrm{~mm}^{3}$. This was followed by randomization of gavage treatment using VE822 or vehicle (Figure 8C). Strikingly, VE822 treatment significantly inhibited tumor growth in both KDM5D-deficient cell lines, whereas this effect was not observed in KDM5D-expressing cell lines (Figure 8D). Collectively, these data indicate that loss of KDM5D can be utilized as a biomarker to predict efficacy of this synthetic lethal approach using an ATR inhibitor.

Clinical impact of KDM5D expression in publicly available data sets. We next assessed the effect of KDM5D expression on out- comes in human PC. We analyzed 2 clinical data sets (NCBI's Gene Expression Omnibus database; GEO GSE21034 and GSE70770) $(9,33,34)$, both of which contain gene expression profiles from radical prostatectomy specimens. Decreased KDM5D expression was significantly correlated with poor clinical outcome in both data sets (Figure 9A). We also found that lower KDM5D expression was associated with a higher likelihood of lethal PC after prostatectomy (odds ratio: 1.61, 95\% CI: 1.03-2.5, $P$ value $=0.04$ ) among 404 men with primary PCs in the Physicians' Health Study and Health Professionals Follow-Up Study (PHS/HPFS) $(35,36)$. A number of data sets revealed that lower KDM5D expression level was associated with higher Gleason score (GS) (>7) in primary PC (Supplemental Figure 7A and refs. 9, 34, 37, 38). Using the CRPC samples from the SU2C cohort (39), we correlated the publicly available mRNA expression data with clinical features of patients treated with the androgen-signaling inhibitors abiraterone and enzalutamide. KDM5D mRNA expression levels were significantly lower in the patients previously treated with abiraterone or enzalutamide than in those with no prior abiraterone or enzalutamide exposure (Supplemental Figure 7B), suggesting that decreased KDM5D expression is progressively lost with progression of CRPC and possibly contributes to resistance to abiraterone and enzalutamide. We also assessed whether decreased KDM5D expression was broadly observed in men with other cancer types. KDM5D mRNA expression levels were significantly lower in cancer specimens compared with normal tissue in various types of cancer, including lung cancer (40), bladder cancer (41), and esophageal cancer (42) (Supplemental Figure 7C). Interestingly, we found that a shorter overall survival in patients with lower KDM5D expression levels was identified in American Joint Committee on Cancer (AJCC) clinical stage 2 or lower, but not in stage 3 or higher in The Cancer Genome Atlas (TCGA; https://cancergenome.nih.gov/) data sets (the data was obtained from the cBioPortal; https://www.cbioportal.org in bladder and colorectal cancer: Supplemental Figure 7D). These data indicate that the KDM5D expression level could be a valuable biomarker for identifying patients with a poor prognosis at a relatively early stage of bladder and colorectal cancer in men. We then sought to examine the correlation between the genes regulated by KDM5D and clinical outcomes. We looked for KDM5D-associated genes that we identified by integrated analysis of RNA-seq and ChIP-seq data from PC cell lines (detailed above; Figure 4) that overlapped with KDM5D-associated genes in human tumors from the Taylor cohort of 150 patients $(9,43)$. In the latter, there were 8,643 genes that were negatively (Pearson's correlation coefficient $\leq 0.3$ ) and 4,533 genes that were positively (Pearson's correlation coefficient $>0.3$ ) correlated with KDM5D expression. Notably, the expression of 69 genes was increased with decreased KDM5D levels (negatively correlated) in both our data from the cell line analysis and human GEP data (Figure 9B and Supplemental Table 4), whereas 11 genes were decreased in both our data and human GEP data when KDM5D was decreased (positively correlated) (Supplemental Figure 7E and Supplemental Table 5). We next explored the clinical significance of the 69 negatively and 11 positively correlated genes in 2 primary PC patient cohorts (TCGA and PHS/HPFS) (35, $36,44)$. In TCGA, higher levels of 60 out of the 69 negatively correlated genes were associated with a poorer DFS, and low levels of 2 out of the 11 positively correlated genes were associated with a 


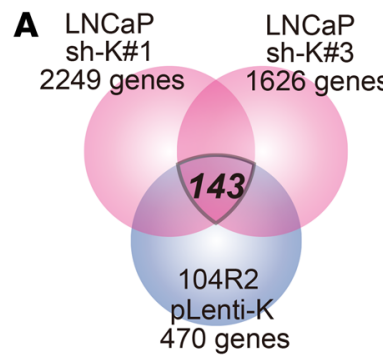

Genes of negative correlation with KDM5D expression level

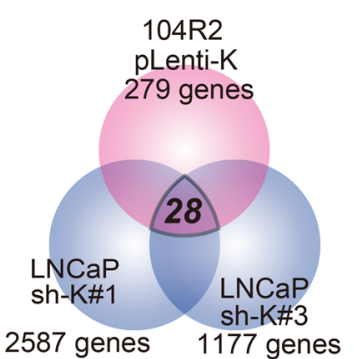

Genes of positive correlation with KDM5D expression leve

Upregulated genes of $\angle$ FDR of 0.01 (vs. control)

Downregulated genes of $<$ FDR of 0.01 (vs. control)

C Top 2 gene sets positively enriched by knockdown of KDM5D in LNCaP

1. DNA replication

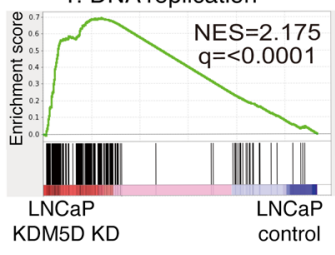

2. Mitotoic M M G1 phases

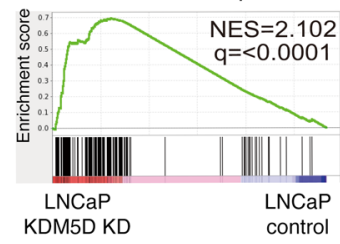

Top 2 gene sets negatively enriched by

overexpression of KDM5D in LNCaP-104R2

1. Mitotic M M G1 phases

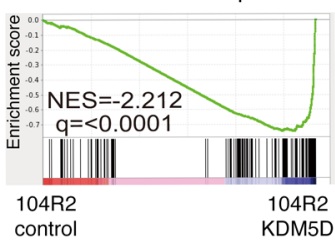

2. DNA replication

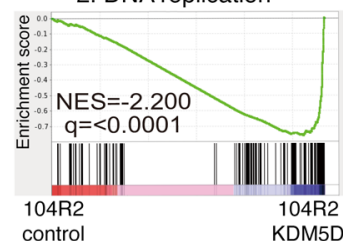

B Low

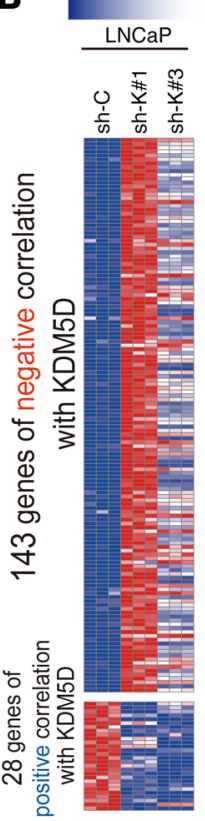

Top 20 gene ontology terms

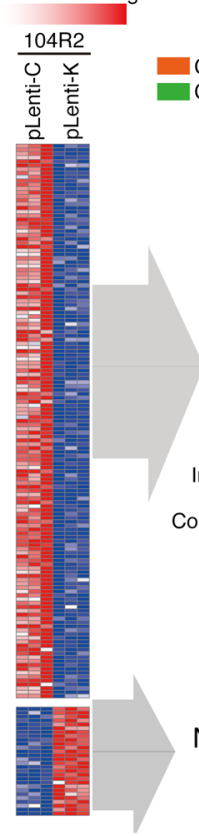

$-\log _{10}$ FDR adjusted $P$ value $\begin{array}{llll}10 & 20 & 30 & 40\end{array}$

GO-biological process component Cell cycle phase Cell cycle process Mitotic cell cycle Mitosis $\mathrm{M}$ phase of mitotic cell cycleCell cycle Organelle fission Cell division
Condensed chromosome
Organelle organization Microtubule cytoskeleton Spindle Intracellular nonmembrane-bound Chromosome centromer Chromosome centromeric region Cytoskeletal part

$$
\text { : }
$$

GO BP DNA replication (FDR: $5.30 \times 10^{-12}$ ) GO BP DNA repair (FDR: $1.10 \times 10^{-2}$ )

None of $\mathrm{GO}$ terms

with FDR $<0.05$
D
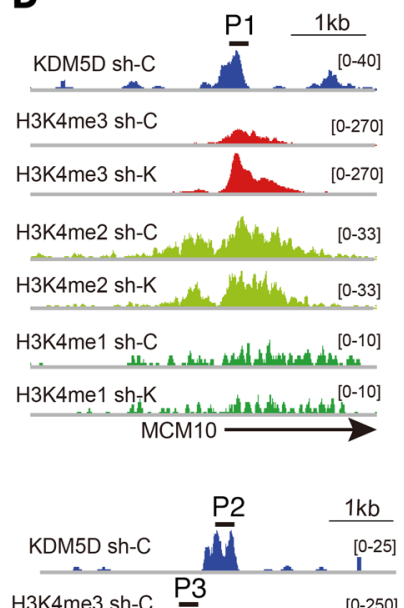

H3K4me3 sh-C [0-250]

H3K4me3 sh-K [0-250]

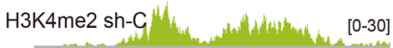

H3K4me2 sh-K [0-30]

$\frac{\mathrm{H} 3 \mathrm{~K} 4 \mathrm{me} 1 \mathrm{sh}-\mathrm{C}}{\mathrm{H} 3 \mathrm{~K} 4 \mathrm{me} 1 \mathrm{sh}-\mathrm{K}}$
MCM10 (minichromosome maintenance 10 replication initiation factor)
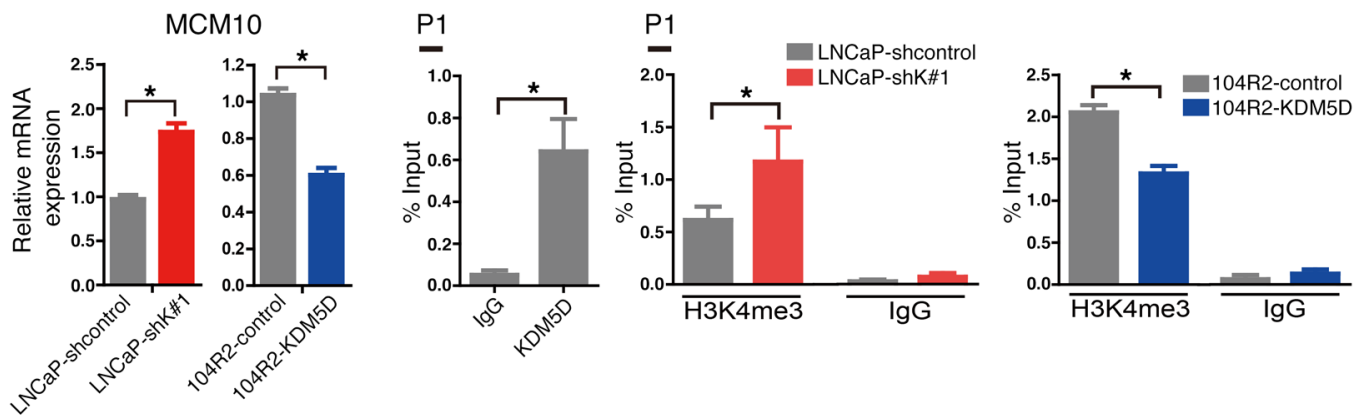

NUF2 (NDC80 kinetochore complex component)
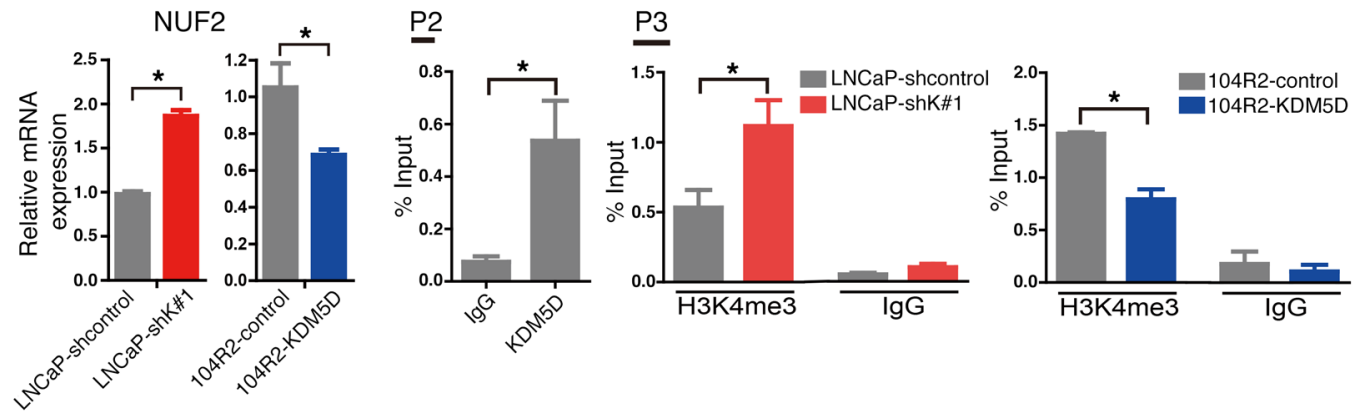
Figure 4. Pathways affected by the modification of KDM5D expression levels. (A) Venn diagram from RNA-seq analysis comparing differentially expressing genes with FDR $<0.01$ in LNCaP sh-control vs. sh-KDM5D\#1, sh-KDM5D\#3, and 104R2 pLenti-control versus pLenti-KDM5D. (B) Heatmap of RNA-seq analysis comparing the differentially expressed genes of 143 negatively collated genes and 28 positively correlated genes with KDM5D expression level. Top $20 \mathrm{GO}$ terms of FDR $<0.05$ in genes of negative correlation are shown. sh-C, control; sh-K, KDM5D. (C) Top 2 positively and negatively enriched pathways by knockdown (in LNCaP sh-control vs. sh-KDM5D\#1) and overexpression (in LNCaP-104R2 control vs. overexpression) of KDM5D, respectively. Pathways were sorted by normalized enrichment score (NES) in GSEA. (D) Peak map of KDM5D and H3K4 methylation marks near the transcription start site of MCM10 and NUF2 in LNCaP sh-control and sh-KDM5D\#1 cells. ChIP-qPCR was performed using primers indicated in peak map, and results of 3 independent experiments are shown as mean $+\mathrm{SD}$. ${ }^{*} P<0.05$, unpaired $t$ test.

poorer DFS (Supplemental Figure 8, A and B). In the PHS/HPFS, higher levels of 43 out of the 69 negatively correlated genes were associated with a significantly higher rate of lethal PC and none were associated with a better outcome (Figure 9C). Lower levels of 4 of the 11 genes positively correlated with KDM5D expression were associated with lethality (Supplemental Figure 8C). In publicly available GEP data from a Mayo Clinic cohort (Illumina DASL Cancer Panel microarray) (45), which included 8 genes out of the 69 negatively correlated genes, higher expression of all 8 genes was associated with shorter cancer-specific survival (Supplemental Figure 9A). To further assess the impact of decreased KDM5D expression in CRPC patients, 2 publicly available data sets were analyzed (refs. 6, 12, and Supplemental Figure 9B). There seemed to be higher levels of the negatively correlated genes and lower levels of the positively correlated genes in CRPC compared with primary cancer, which further suggested decreased KDM5D expression as a driver of CRPC. Finally, we clustered 131 primary PC patients in the Taylor cohort (9) based on expression levels of 69 negatively correlated genes, and 14 patients were identified as the KDM5D loss signature(Figure 9D). As expected, KDM5D expression levels were significantly lower in the KDM5D loss signature (Figure 9E), and these patients had a significantly shorter DFS (Figure 9F). Taken together, these data show that decreased KDM5D expression and the cluster of genes downstream substantially affect clinical outcome in PC patients as well as other cancer types in males.

\section{Discussion}

The biology, response to ADT, and gene expression profile of primary hormone-naive PC and CRPC are distinctly different (39, 44 ), yet the molecular basis for this is not well understood. Given the heterogeneity of response to primary ADT, it is possible that the de novo presence of biological drivers may exist early in the few patients who develop early CRPC, while in others, these drivers may evolve over time, resulting in a longer time to development of CRPC. With regard to the bioinformatic analysis, the enrichment in repetitive and palindromic sequences on the $\mathrm{Y}$ chromosome makes studies of CNAs on the $\mathrm{Y}$ chromosome challenging. In the present study, we demonstrated deletion of KDM5D on the Y chromosome in CRPC LNCaP-104R2 cells, whereas most parent LNCaP cells have more than 2 copies of the $\mathrm{Y}$ chromosome, with a small fraction of the cells having loss of the KDM5D locus. These data suggest that with ADT, the subpopulation of LNCaP cells with deletion of KDM5D may survive preferentially, which results in the acquisition of a more aggressive phenotype resulting from progression out of cell-cycle arrest and DNA-replication stress. Although it is still unclear whether these subpopulations with the deletion are of clonal origin, the data from the TMA suggest that decreased expression of KDM5D may at least be in some cases due to partial or large deletions of the Y chromosome. Since the extent of the deletion of KDM5D seemed to be broad enough to allow for detection of loss of KDM5D in patients with primary PC, it is plausible that KDM5D loss may be assessed by FISH analysis.

At a mechanistic level, we showed that loss of KDM5D leads to aberrant $\mathrm{H} 3 \mathrm{~K} 4 \mathrm{me} 3$ active transcriptional marks, resulting in DNA-replication stress. It is clear that these features are hallmarks of aggressive cancer $(18,30)$ and possibly cause genomic instability (31) and genomic alteration; these features have been clearly identified with disease progression in PC (39). Moreover, the commercially available prognostic gene signatures, which often include cell-cycle genes (46) and genes associated with the cell-cycle progression (CCP) score developed by the Transatlantic Prostate Group, which consists of mRNA expression levels of 31 cell-cycle-related genes (47), were substantially similar to our set of genes. Notably, we found higher levels of $\mathrm{G}_{2} / \mathrm{M}$ checkpoint factors, including CDK1 and CDC25C, with the loss of KDM5D as well as concomitant ATR activation by DNA-replication stress. Given that activated ATR signaling, which prevents stalled fork collapse by replication stress, leads to $\mathrm{G}_{2} / \mathrm{M}$ arrest as a consequence of the inhibition of CDC25C-cyclin B1/CDK1 activity (26, 27), it is possible that the cells that undergo loss of KDM5D harbor an aggressive property, with the replication stress bypassing the $\mathrm{G}_{2} / \mathrm{M}$ arrest activating mediators such as CDK1 and CDC25C.

Toledo et al. first showed that ATR inhibitors elicit the breakage of stalled replication forks and that the combination use of hydroxyurea, which promotes fork stalling due to dNTP depletion, leading to DNA-replication stress, synthetically creates double-strand break (48). This synthetic lethality exploiting stress sensitization by the ATR inhibitor has been recognized as having a new therapeutic potential with the combination use of other DNA-damaging agents in various type of cancers $(28,49,50)$. In the current study, we showed that cells with DNA-replication stress induced by loss of KDM5D were particularly sensitive to an ATR inhibitor (stress sensitization) showing increased DNA damage. This indicates that detecting loss of KDM5D could serve as a biomarker predicting the efficacy of ATR inhibition, which may help select patients for this new targeted therapy. Finally, it is known that the $\mathrm{Y}$ chromosome is frequently lost in peripheral blood cells with advancing age in men and that this loss is associated with nonhematological cancer-specific mortality (51, 52); however, the mechanism by which these findings biologically occur is unknown. The present study revealed an epigenetic role of male-specific histone demethylase KDM5D encoded on the Y chromosome in PC cells. Further study is warranted to enable development of a strategy wherein use of an ATR inhibitor could also affect the peripheral blood cells with loss of the $\mathrm{Y}$ chromosome.

\section{Methods}

Cell lines, proliferation assay. The PC cell lines (LNCaP, PC3) were obtained from ATCC. Authentication was obtained by a Human 
A
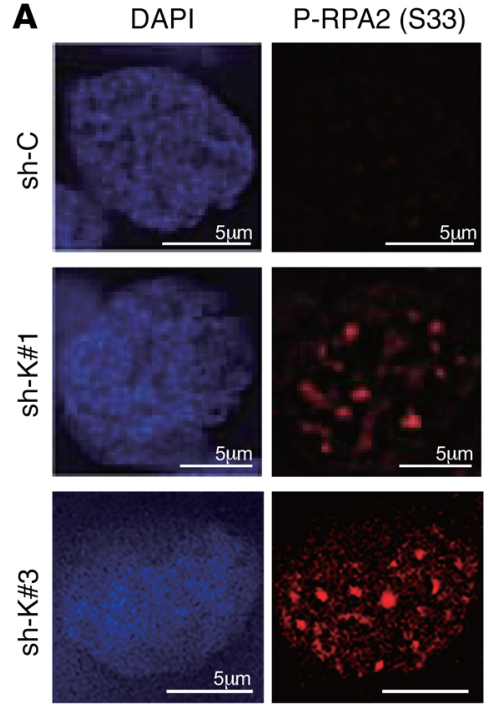

B

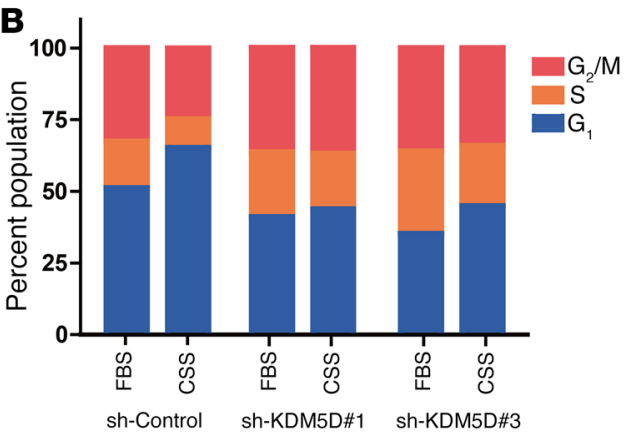

sh-KDM5D\#1 sh-KDM5D\#3
Merge
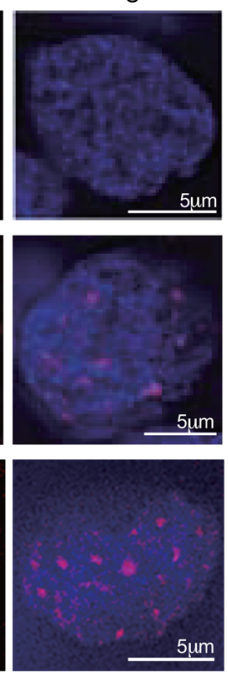

DAPI
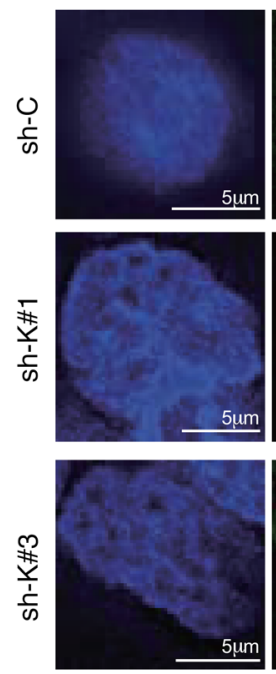

C
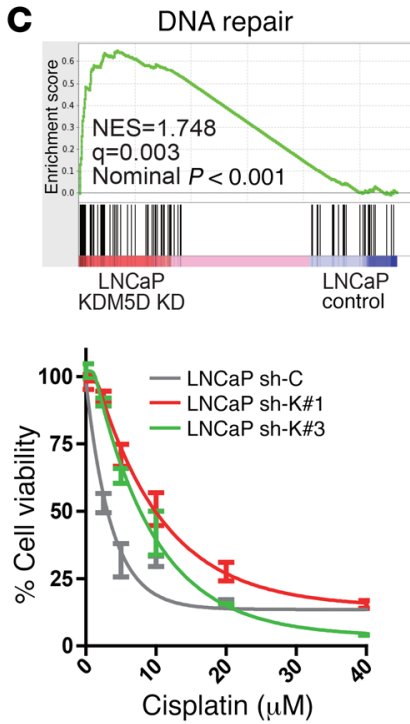

$\mathrm{rH} 2 \mathrm{AX}$
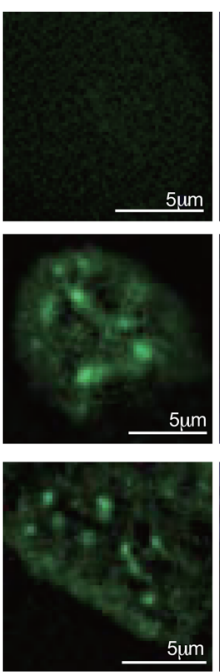

Merge
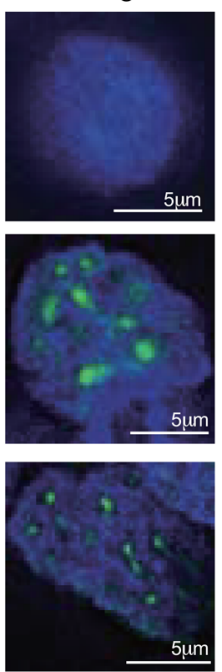
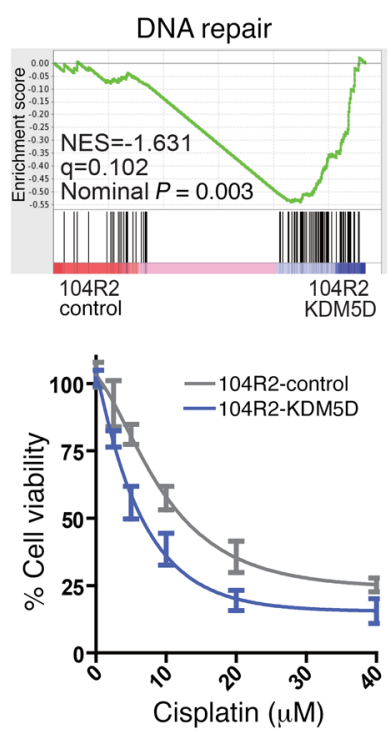

Figure 5. Aberrant DNA damage during S phase leading to DNA-replication stress. (A) LNCaP sh-control, sh-KDM5D\#1, and sh-KDM5D\#3 cell lines were stained by immunofluorescence for replicative stress markers, including p-RPA2 and p-rH2AX. sh-K\#1, sh-KDM5D\#1. (B) Cell-cycle analysis in LNCaP sh-control, sh-KDM5D\#1, and sh-KDM5D\#3. Cells were cultured in FBS or CSS medium for 3 days, then collected for analysis. (C) CSEA enrichment profiles in DNA_Repair pathway (MSigDB: M18229) by knockdown (in LNCaP sh-control vs. sh-KDM5D\#1) and overexpression (in LNCaP-104R2 control vs. overexpression) of KDM5D, respectively. Cell-growth assay was used to evaluate effect of cisplatin in indicated cell lines after 96 hours of treatment. The inhibitory effect on cell growth by cisplatin is presented as a relative value (mean $\pm \mathrm{SD}$ ) compared with control as $100 \%$.

STR Profiling Cell Authentication Service (ATCC), and mycoplasma was tested in all cell lines. The LNCaP-C42 cell line was obtained from ViroMed Laboratories. The LNCaP-104R2 cell line was provided by Shutsung Liao (University of Chicago, Chicago, Illinois, USA), and the E006AA cell line was provided by John T. Isaacs (The Johns Hopkins University School of Medicine, Baltimore, Maryland, USA). These cells were maintained in 10\% FBS (LNCaP, LNCaP-C42, PC3, and E006AA) or 10\% CSS (LNCaP-104R2) supplemented with $2 \mathrm{mM}$ L-glutamine at $37^{\circ} \mathrm{C}$ in $5 \% \mathrm{CO}_{2}$. Cells treated in individual experiments were assessed for cell viability using CellTiter-Glo Luminescent Assay (Promega) following the manufacturer's protocol by incubating cells in a 96-well format in 1:1 media/luminescent reagent for 10 minutes.

Soft agar colony formation assay. Live cells (50,000 LNCaP sh-control, sh-KDM5D\#1, and sh-KDM5D\#3) were mixed with $0.3 \%$ top-agar and were plated onto $0.6 \%$ base-agar in 6-well plates with triplicates. The cells were incubated for 21 days, changing the CSS medium with $0.15 \mu \mathrm{g} / \mathrm{ml}$ doxycycline every 3 days, followed by $0.1 \%$ iodonitrotetrazolium chloride (INT) staining overnight. The plates were photographed, and the colony numbers were counted by GelCount (Oxford Optonix).

FISH. Cells were treated with Colcemid at a final concentration of $0.1 \mu \mathrm{g} / \mathrm{ml}$. Following 30 to 60 minutes incubation, the cells were trypsinized, resuspended in $0.075 \mathrm{M} \mathrm{KCl}$, incubated for 10 minutes at $37^{\circ} \mathrm{C}$, and then fixed in methanol-acetic acid (3:1). FISH analysis was performed on the fixed cells using a 3-color KDM5D/Yp11/ CenX probe mix. The probe mix consisted of a BAC clone containing the full-length KDM5D (Yq11) gene (RP11-188C1 and RP11-204P21; labeled with Red dUTP), a BAC clone spanning the Yp11 locus (RP11- 
A

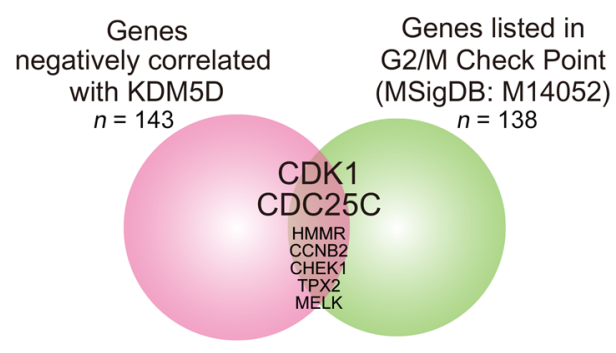

B

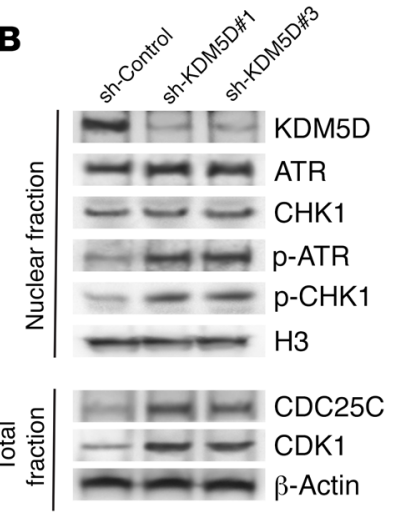

D

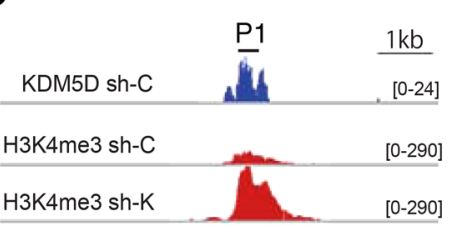

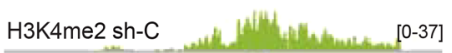

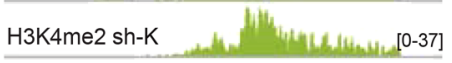

H3K4me1 sh-C ath dit the [0-10]

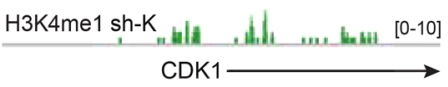
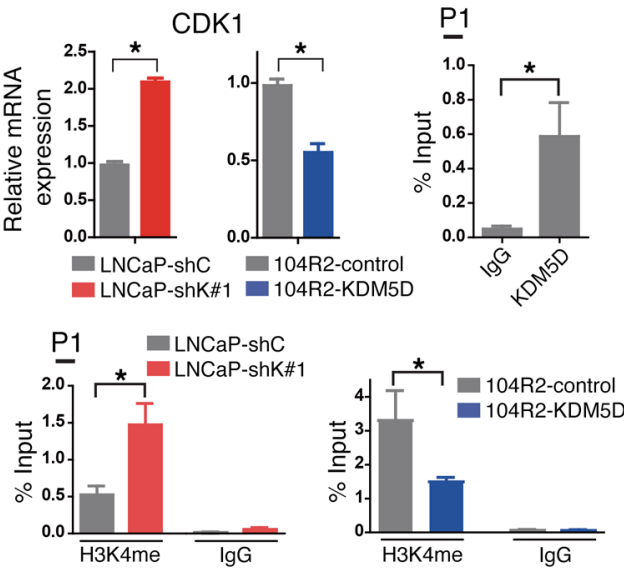

E
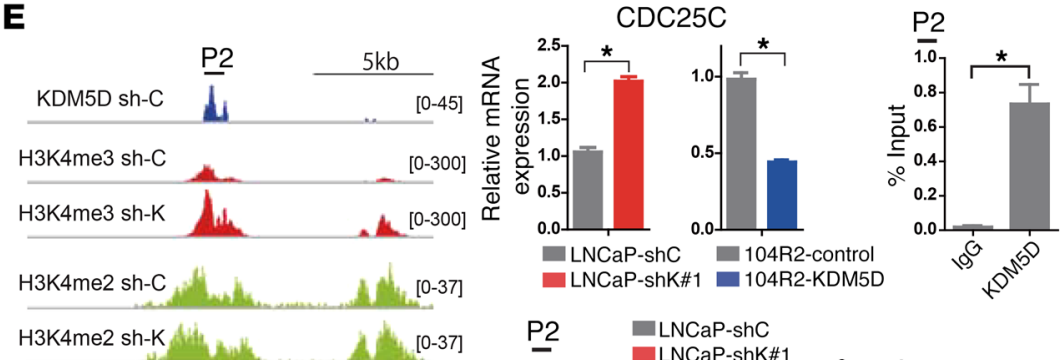
LNCaP-shK\#1 104R2-KDM5D

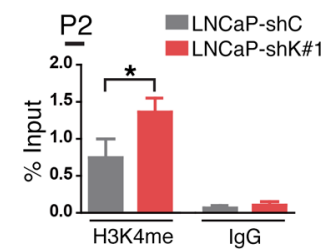

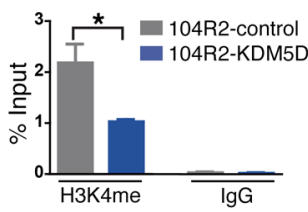

C
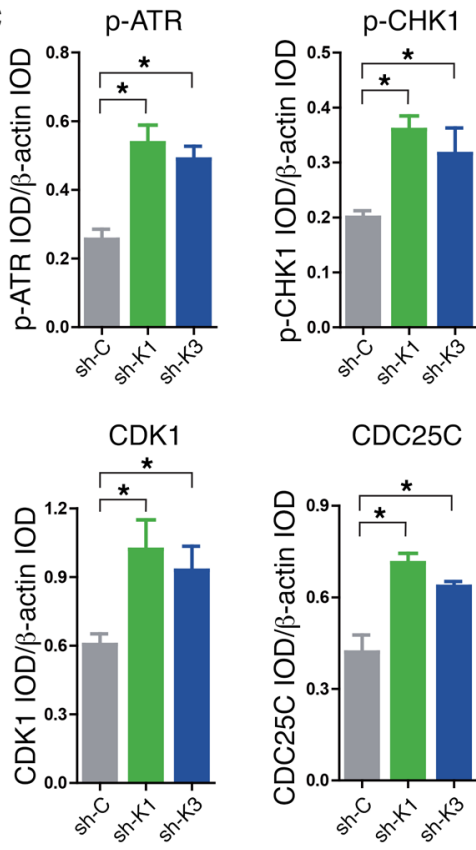

CDC25C

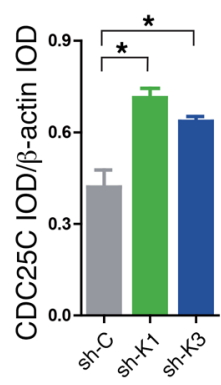

F

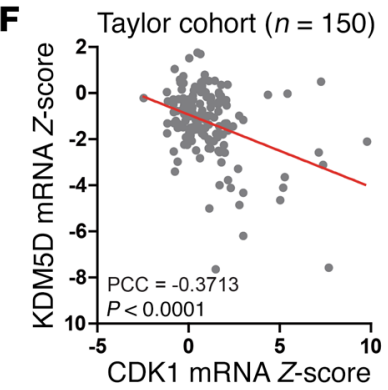

Taylor cohort $(n=150)$

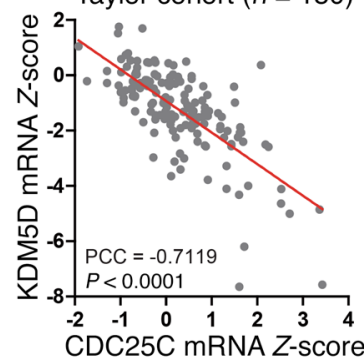

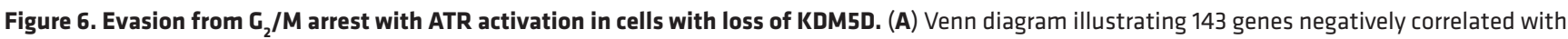
KDM5D expression and 138 genes listed in $G_{2} / M$ checkpoint pathway (MSigDB systematic name, M14052). (B) Nuclear fraction or total cell lysate was collected in indicated cell lines and subjected to immunoblotting using indicated antibodies, including KDM5D, ATR, p-ATR, CHK1, p-CHK1, H3 (loading control), CDK1, CDC25C, and bactin (loading control). (C) Quantitative evaluation of the result from immunoblotting in p-ATR, p-CHK1, CDK1, and CDC25C. Integrated optical density (IOD) was determined in 3 independent experiments for LNCaP sh-control, sh-KDM5D\#1, and sh-KDM5D\#3, and results from 3 independent experiments are shown as mean $+\mathrm{SD}$. ${ }^{*} P<0.05$, unpaired $t$ test. sh-K1, sh-KDM5D\#1. (D and E) Peak map of KDM5D and H3K4 methylation marks near the transcription start site of CDK1 and CDC25C in LNCaP sh-control and sh-KDM5D\#1 cells. ChIP-qPCR was performed using primers indicated in peak map, and results from 3 independent experiments are shown as mean $+S D$. ${ }^{*} P<0.05$, unpaired $t$ test. (F) Correlation of mRNA expression levels between KDM5D and CDK1 (upper panel) and KDM5D and CDC25C (lower panel) in the Taylor cohort $(n=150)(9)$. PCC, Pearson's correlation coefficient.

126H13 and RP11-1125G23; labeled with green dUTP), and a centromeric repeat plasmid specific to the $\mathrm{X}$ chromosome (CenX) (pSV2x5; labeled with Orange dUTP). The Yp11 control probe was included to detect and distinguish loss of KDMD5 due to deletions versus the loss of the entire Y chromosome. CenX probe served as the control for hybridization (in situations with complete loss of Y chromosome, 0 copies). Probe labeling, tissue processing, hybridization, posthybridization washing, and fluorescence detection were performed 

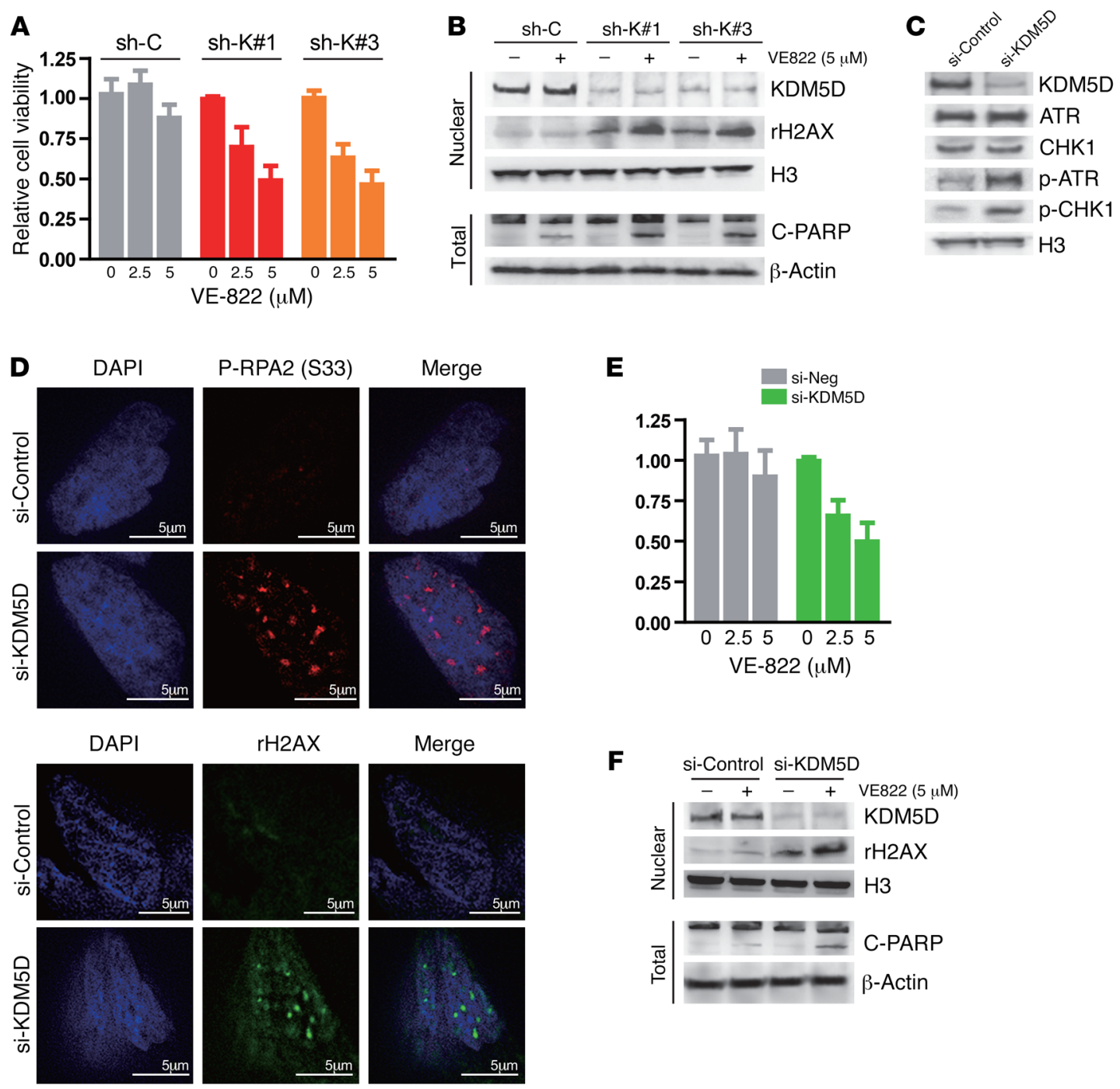

$\mathbf{F}$

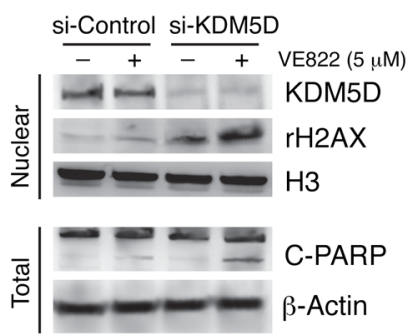

Figure 7. Synthetic lethal approach exploiting DNA-replication stress by the loss of KDM5D. (A) Data on growth of cells treated with VE822 in LNCaP sh-control, sh-KDM5D\#1, and sh-KDM5D\#3. Cells were treated with the indicated concentrations of VE822 for 72 hours. The inhibitory effect on cell growth is presented as a relative value (mean \pm SD) compared with control as $100 \%$. The results of 3 independent experiments are shown. (B) Nuclear fractions and total cell lysates were collected in LNCaP sh-control, sh-KDM5D\#1, and sh-KDM5D\#3 after 24 hours of treatment with DMSO or $5 \mu$ M VE822 and subjected to immunoblotting with the indicated antibodies. (C) Nuclear fraction was collected in VCaP cells with si-control and si-KDM5D and subjected to immunoblotting using indicated antibody including KDM5D, ATR, p-ATR, CHK1, p-CHK1, and H3 (loading control). (D) VCaP cell line with or without siKDM5D was stained by immunofluorescence for the replicative stress markers, including p-RPA2 and p-rH2AX. (E) Data on growth of cells treated with VE822 in VCaP cells using si-control or si-KDM5D. Cells were treated with the indicated concentrations of VE822 for 72 hours. The inhibitory effect on cell growth is presented as a relative value (mean \pm SD) compared with control as $100 \%$. The results of 3 independent experiments are shown. (F) Nuclear fractions and total cell lysates were collected in VCaP cells using si-control or si-KDM5D after 24 hours of treatment with DMSO or $5 \mu$ M of VE822, and subjected to immunoblotting with the indicated antibodies.

according to standard laboratory procedures. Slides were scanned using a Zeiss Axioplan 2i epifluorescence microscope equipped with a megapixel CCD camera (CV-M4+CL, JAI) controlled by Isis 5.5.9 imaging software (MetaSystems Group Inc.). In cell lines, a minimum of 200 intact consecutive nuclei and 20 consecutive metaphases were scored to characterize loss of KDM5D and assess heterogeneity. For TMAs (T195c and PR1921a; purchased from US Biomax), a minimum of 50 to 100 nuclei per case were evaluated. To minimize truncation artifacts, only nuclei with at least 1 control signal were considered. A case was considered to exhibit loss of KDM5D if more than $90 \%$ of tumor cells in a minimum of approximately 2 to 5 adjacent or non- adjacent fields $(\times 63)$ showed absence of red (KDM5D) signal. A case was considered to exhibit intratumoral heterogeneity when a distinct topographic region or regions within a core were uniformly KDM5D positive or uniformly KDM5D negative. Heterogenous ploidy coupled with truncation artifacts precluded reliable assessment and/or quantification of intratumoral heterogeneity when intratumoral heterogeneity was present as a mixed population of KDM5D-positive and KDM5D-negative cells in the given region.

Cell-cycle analysis. LNCaP-sh-control and sh-KDM5D\#1 cells were cultured in $10 \%$ FBS or CSS medium for 72 hours, and 1 million cells were harvested, followed by washing with HBSS and fixing 
A

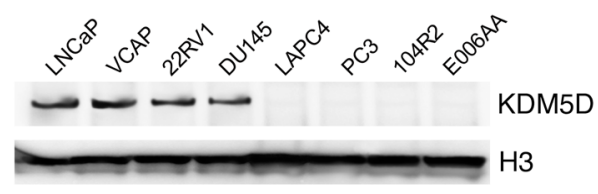

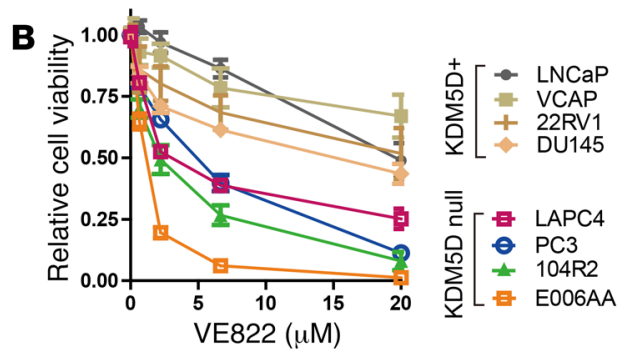

C

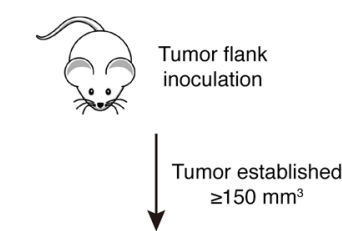

Treatment randomization

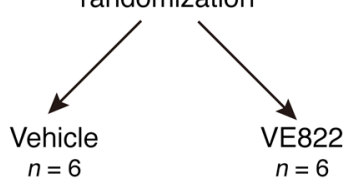

D

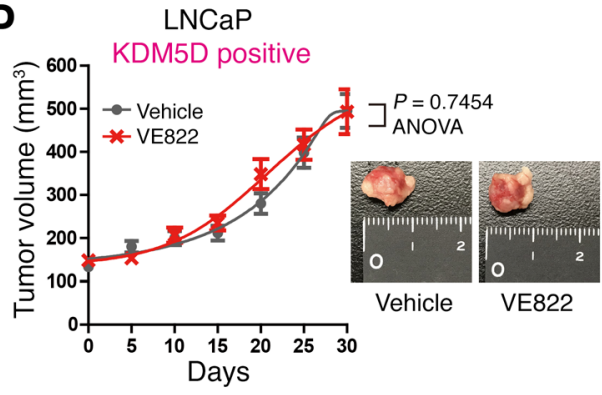

LNCaP-104R2

KDM5D negative

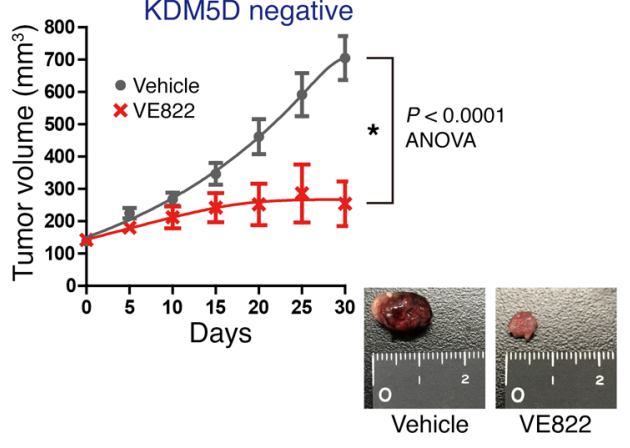

22RV1

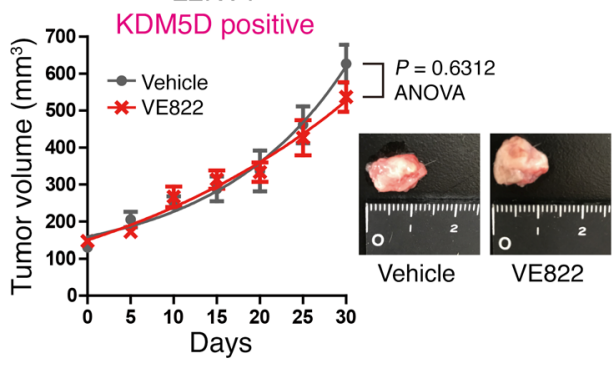

E006AA

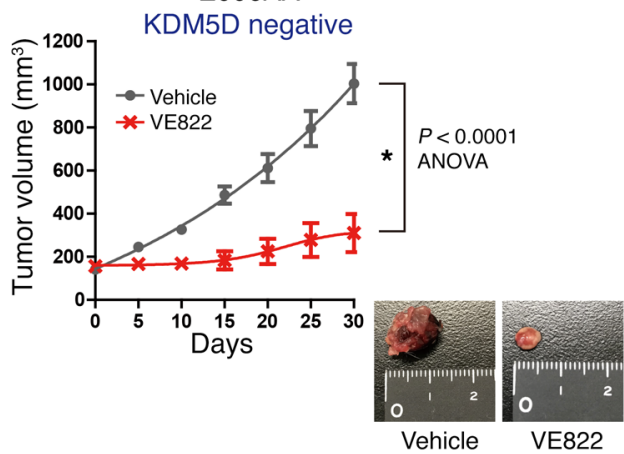

Figure 8. Higher sensitivity of ATR inhibitor to PC cells deficient in KDM5D in an in vivo xenograft mouse model. (A) Nuclear fraction was collected in indicated PC cell lines and subjected to immunoblotting using KDM5D and H3 (loading control) antibodies. (B) PC cell lines were treated with the indicated concentrations of VE822 for 72 hours. The inhibitory effect on cell growth by VE822 is presented as a relative value (mean \pm SD) compared with control as $100 \%$. The results of 3 independent experiments are shown. (C) Schematic representation of the protocol for xenograft mouse model. After tumors developed to a volume of $150 \mathrm{~mm}^{3}$, mice were randomized into 2 groups (vehicle or VE822 treatment) with 6 mice in each group. Treatment was administered 4 times weekly for 4 weeks. (D) Tumor growth of KDM5D-positive (LNCaP and 22RV1) and KDM5D-deficient (LNCaP-104R2 and E006AA) cells in xenograft mouse model treated with VE822 (60 mg/kg, 4 times weekly) or vehicle. Representative images of tumors in each cell line are shown. ${ }^{*} P<0.05$, ANOVA.

with ice-cold $70 \%$ ethanol overnight. The cells were then washed with HBSS and stained with $200 \mu \mathrm{PI}$ /RNAse reagent (Millipore) for 30 minutes. Distribution of the cell cycle was analyzed by the Muse Cell Analyzer (Millipore).

qPCR. RNA was isolated using TRIzol (Invitrogen) and Directzol RNA Prep Plus (Zymo Research) according to the manufacturer's protocol, followed by quantification using a NanoDrop spectrophotometer, and $1 \mu \mathrm{g}$ RNA was reverse-transcribed using a High Capacity cDNA Reverse Transcription Kit (Applied Biosystems). The primers used are listed in Supplemental Table 10.

qPCR was performed on an ABI QuantStudio 5 detector (Thermo Fisher Scientific). Product formation was detected by incorporation of SYBR Green I using ROX as a passive reference. The expression data were normalized with GAPDH in each sample. Experiments were repeated and analyzed 3 times.

Immunoblotting and cell fractionation. Whole-cell lysates were collected and lysed in radioimmunoprecipitation assay (RIPA) lysis buf- fer with proteinase inhibitor mixture (Thermo Scientific) and sonicated using a Bioruptor Standard (Diagenode) for 5 minutes. For cellular protein fractionation, hypotonic lysis buffer $(50 \mathrm{mM}$ Hepes- $\mathrm{NaOH}$, pH 7.5, 10\% glycerol, 0.5\% Nonidet P-40, 0.25\% Triton X-100, proteinase inhibitor mixture) was used for extracting cytoplasmic proteins. Nuclear pellets were washed with cold PBS once and dissolved in high-salt nuclear extraction buffer $(0.1 \%$ SDS, $10 \mathrm{mM}$ Tris $\cdot \mathrm{HCl}$, $150 \mathrm{mM} \mathrm{NaCl}, 0.1 \%$ Triton-X, proteinase inhibitor mixture) and sonicated using a Bioruptor Standard for 5 minutes, followed by gentle agitation for 30 minutes at $4^{\circ} \mathrm{C}$. After centrifugation at $16,000 \mathrm{~g}$ for 5 minutes, supernatant was collected as nuclear fractions. Proteins were subjected to NuPAGE Bis-Tris Gels or NuPAGE Tris-Acetate Gels before being transferred onto PVDF membrane (Millipore). The list of antibodies is provided in Supplemental Table 11. Detection of the protein was performed using the FUSION FX imaging system, and the FUSION-CAPT Advance analyzing system was employed for the quantification of the protein level. 

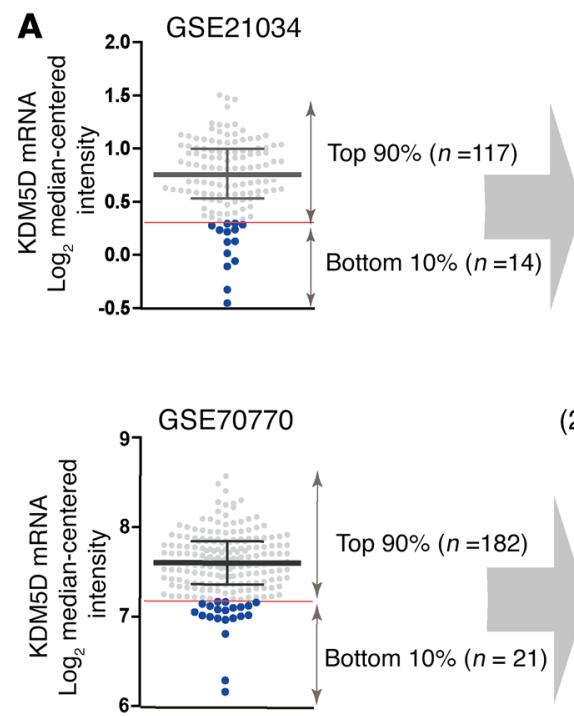

Taylor cohort (131 primary cancer from prostatectomy)

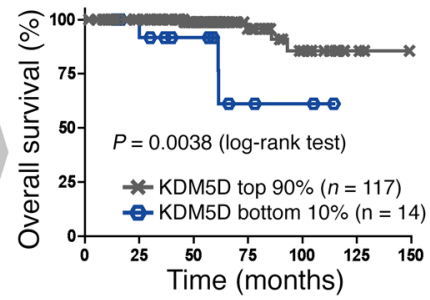

Whitington cohort

(203 primary cancer from prostatectomy)

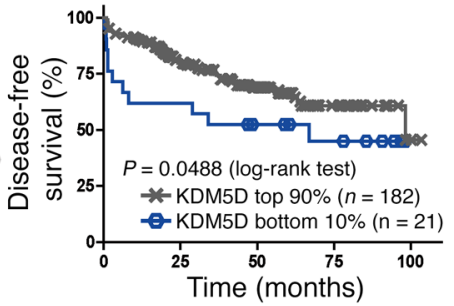

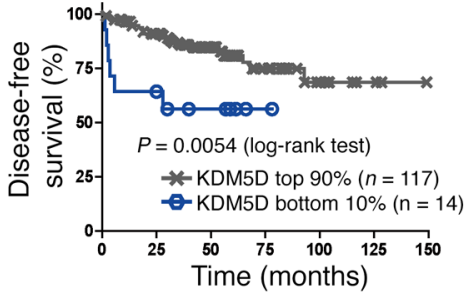

B

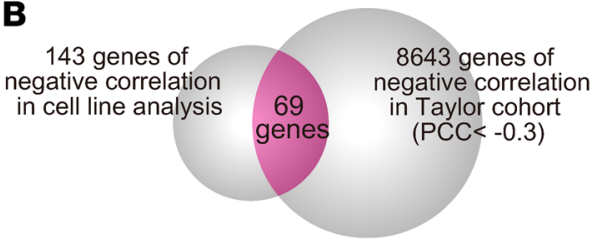

C PHS/HPFS (404 primary prostate cancer)

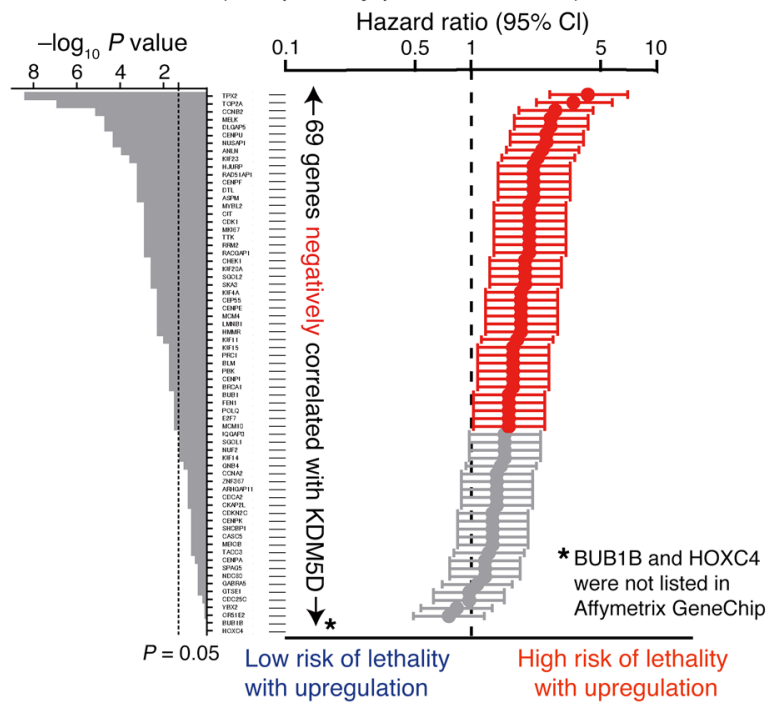

$\mathbf{E}$

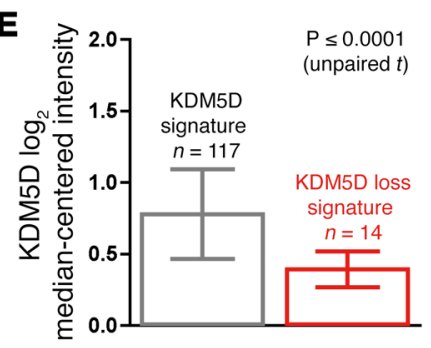

$\mathbf{F}$

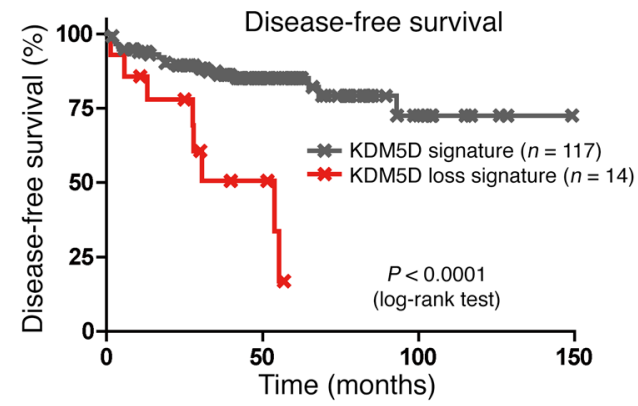

D

Relative

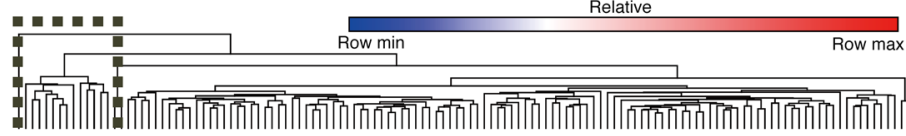

Patient no. 1.

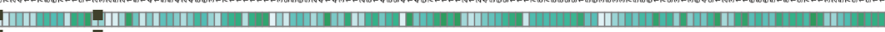

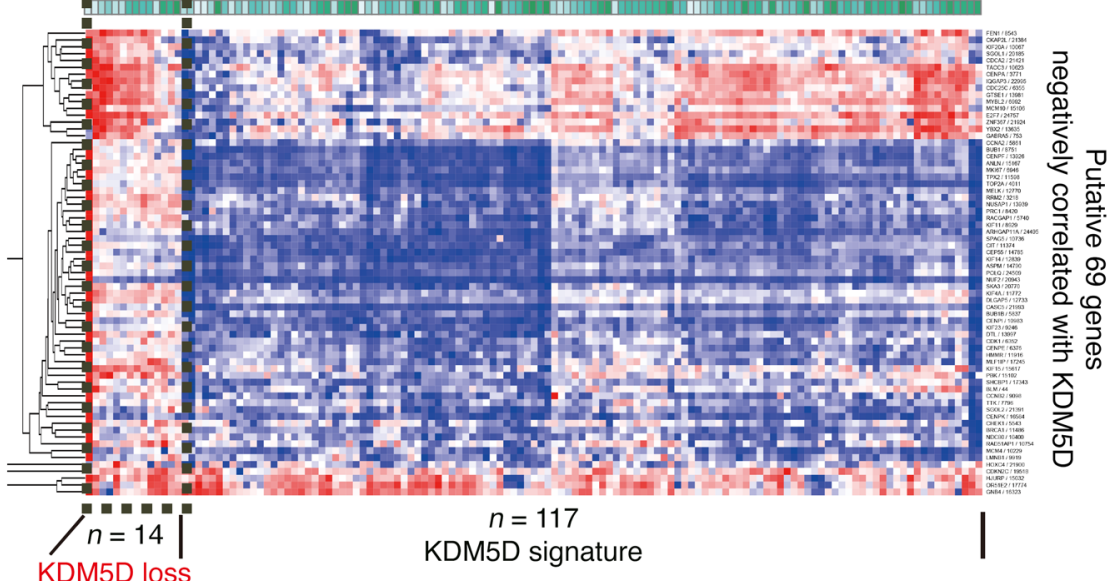

signature 
Figure 9. Clinical impact of KDM5D expression in publicly available data sets. (A) Clinical significance of KDM5D expression level. Patients in 2 cohorts (Taylor cohort, ref. 9; Whitington cohort, refs. 33, 34) were divided into 2 groups (top 90th percentile and bottom 10th percentile), and Kaplan-Meier curves were defined in each group. The log-rank test was calculated to determine significance. (B) Venn diagram showing genes negatively correlated with KDM5D expression levels in cell line analysis (143 genes) and human GEP data in the Taylor cohort (9). (C) Hazard ratio for the lethality in 404 PHS/HPFS PC patients $(35,36)$ among 69 genes negatively correlated with KDM5D expression levels. Patients were divided by the median expression of each genes and analyzed using univariate analysis. (D) Hierarchical clustering of 131 primary patients in the Taylor cohort according to 69 genes negatively correlated with KDM5D expression levels, illustrating 14 patients with KDM5D loss signature. (E) DM5D mRNA expression levels between KDM5D loss signature and KDM5D signature patients. Unpaired $t$ test was used to determine significance. (F) Kaplan-Meier curve demonstrating DFS in KDM5D and KDM5D loss signature. The log-rank test was used to determine significance.

RNA-seq. PolyA $\mathrm{A}^{+} \mathrm{RNA}$ was purified using a polyA Spin mRNA Isolation Kit (New England Biolabs), followed by library preparation for 40 ng of purified RNA. RNA fragmentation, first- and second-strand cDNA synthesis, and end-repair processing were performed using the NEBNext Ultra RNA Library Prep Kit for Illumina (New England Biolabs). The adaptor was ligated to the fragments for multiplex samples using NEBNext Multiplex Oligos for Illumina index primers (New England Biolabs), and the libraries were amplified by 14 cycles of PCR. The products were size fractionated by running on an $8 \%$ polyacrylamide gel, and final libraries were purified from the gel. Fragment sizes were validated by using a High Sensitivity DNA Kit (Agilent Technologies) on an Agilent 2100 Bioanalyzer. Biological triplicates were sequenced by Illumina NextSeq 500 (SR75) at the Dana-Farber Cancer Institute Center for Cancer Computational Biology Core Facility.

ChIP-seq. Cells $\left(7.5 \times 10^{6}\right)$ at room temperature were crosslinked for 10 minutes with $1 \%$ paraformaldehyde (PFA), and the reaction was terminated by the addition of $1 \mathrm{ml} 1.25 \mathrm{M}$ glycine for 5 minutes, followed by extracting the nuclear fraction using the hypotonic lysis buffer described in the cell fractionation protocol. Crosslinked chromatin was transferred to AFA fiber tubes (Covaris) and sonicated to an average fragment size of 200-300 bp in 0.2\% SDS shearing buffer using a Covaris sonicator. Sonicated chromatin was centrifuged for 5 minutes at $16,000 \mathrm{~g}$ and diluted to $0.1 \%$ SDS concentration. After preclearing with Dynabeads Protein $\mathrm{G}$ (Life Technologies) for 1 hour, chromatin-protein complexes were immunoprecipitated with $5 \mu \mathrm{g}$ antibodies overnight at $4^{\circ} \mathrm{C}$. The next day, Dynabeads Protein $\mathrm{G}$ was added for 2 hours, and beads were washed with the following buffers: low-salt wash buffer (20 mM Tris $\cdot \mathrm{HCl}, \mathrm{pH} 8.0,150 \mathrm{mM} \mathrm{NaCl}, 0.1 \%$ SDS, $1 \%$ Triton $\mathrm{X}-100$, $2 \mathrm{mM}$ EDTA), high-salt wash buffer $(20 \mathrm{mM}$ Tris $\cdot \mathrm{HCl}, \mathrm{pH} 8.0,500 \mathrm{mM}$ $\mathrm{NaCl}, 0.1 \%$ SDS, 1\% Triton X-100, 2 mM EDTA), LiCl wash buffer (10 $\mathrm{mM}$ Tris $\cdot \mathrm{HCl}, \mathrm{pH}$ 8.0, $250 \mathrm{mM} \mathrm{LiCl}$, 1\% IGEPAL CA-630, 1\% sodium deoxycholate, $1 \mathrm{mM}$ EDTA), and TE buffer. Precipitated chromatin was then eluted from the beads in $300 \mu$ l elution buffer (1\% SDS, $0.1 \mathrm{M}$ $\mathrm{NaHCO}_{3}$ ) for 1 hour at room temperature followed by decrosslinking at $65^{\circ} \mathrm{C}$ overnight. After RNase A and proteinase $\mathrm{K}$ treatment, ChIP and input DNA were extracted by phenol-chloroform extraction. Fragment sizes (200-300 bp) were evaluated by a High Sensitivity DNA Kit (Agilent Technologies) on an Agilent 2100 Bioanalyzer. The specific enrichment was analyzed by qPCR and percentage of input calculation. For ChIP-seq library preparation, the ThruPLEX DNA-seq Kit (Rubicon
Genomics) was used with 2 ng DNA input in 10 cycles of PCR amplification. The products were size fractionated and purified by a polyacrylamide gel, and fragment sizes and library concentrations were validated as above prior to sequencing reactions. Purified libraries were then sequenced by Illumina HiSeq 2500 (PE50) at the Memorial Sloan Kettering Cancer Center Integrated Genomics Operation Core Facility. ChIP-seq raw data were mapped by Bowtie using default parameters to hg19 (Human Genome version 19: NCBI build).

RNA interference, DNA transfection, and lentiviral transduction. Sequences of shRNAs used are listed in Supplemental Table 12. Mammalian Gene Collection human KDM5D sequence-verified cDNA (BC144102) was purchased from Dharmacon. Individual shRNAs were designed using Enhanced Direct for Licensees (rnai.co.jp/lsci/ e-sidirect.html) considering mismatch potential of greater than 0.3 and longest common factor (LCF) of less than 9. siRNA transfections targeting KDM5D (ON-TARGET plus siRNA) were purchased from Dharmacon (catalog L-010820-00-0005) and performed using Lipofectamine RNAiMAX (Invitrogen). Twenty-four hours before transfection, cells were seeded onto 6-well plates. The cells were transfected with $50 \mathrm{nM}$ siRNA as described in the manufacturer's protocol and maintained for 48 hours, followed by the designed experiments. For lentiviral transduction, pLKO-Teton-puro, pLenti-CMyc-DDK-IRESGFP, and pHAGE PGK-GFP-IRES-LUC-W vectors were transfected with psPAX2 packaging and pMD2.G envelope plasmid to HEK293FT cells for 2 days using Lipofectamine 3000 (Invitrogen). Thereafter, PC cells were infected with viral supernatants (filtered through a 0.45 $\mu \mathrm{m}$ filter) in the presence of $8 \mu \mathrm{g} / \mathrm{ml}$ polybrene. For shRNAs, a spininfection protocol was applied using 6-well plates at 16,000 $g$ for 60 minutes (Heraeus Multifuge X1 Centrifuge Series, Thermo Scientific), followed by incubation at $37^{\circ} \mathrm{C}$. The next day, medium was changed to fresh medium, and the cells transduced with virus were incubated for 3 days, followed by selection using puromycin (1-1.5 ng/ml).

Immunofluorescence staining. LNCaP sh-control, sh-KDM5D, and VCaP cells were plated onto a collagen-coated 24-well culture dish for 3 days, fixed in 4\% PFA for 10 minutes, washed 3 times with HBSS, and incubated with $0.1 \mathrm{M}$ glycine for 10 minutes to quench PFA autofluorescence. After washing again, cells were permeabilized and stained with a solution of $0.1 \%$ Triton $\mathrm{X}-100$ and HBSS plus BSA $1 \%$, containing primary antibodies at a ratio of 1:250. Cells were washed and incubated for 45 minutes with appropriate secondary fluorescent antibodies. Alexa Fluor 488 anti-rabbit and Alexa Fluor 488 and Alexa Fluor 568 anti-mouse antibodies were purchased from Invitrogen (Life Technologies). After washes, the nuclear content was stained with DAPI reagent (Invitrogen, Life Technologies) overnight at $-20^{\circ} \mathrm{C}$.

In vivo experiments. NOD/SCID mice (NOD.CB17-Prkdcscid/J: Charles River) were anesthetized using isoflurane, and tumor inoculation was performed under surgically sterile conditions. For the orthotopic PC xenograft model, the abdomen was cleaned with iodine solution and a $1 \mathrm{~cm}$ midline incision was created to expose the prostate gland. A 28-gauge needle and a $1 \mathrm{ml}$ disposable syringe were used for injection of the cell suspension. The cells planned for inoculation were prepared at 2.5 million cells in $50 \mu \mathrm{PBS}$ and then mixed with 50 $\mu l$ Matrigel (Corning Matrigel Matrix High Concentration) in a total $100 \mu \mathrm{l}$ cell suspension. The needle was inserted into the dorsal lobe of the prostate, and $100 \mu \mathrm{l}$ of cell suspension was carefully inoculated so as not to have any leakage to the surrounding region. The abdominal wound was closed in 2 layers with a 6/0 absorbable surgical suture. Sur- 
gical castration was performed 2 weeks after the inoculation. A $0.5 \mathrm{~cm}$ linear scrotal incision was made, and both sides of the muscular sacs containing the testes were exposed. After the isolation of vessels and vas deferens, both testes were removed and the wound was closed with $6 / 0$ absorbable surgical suture. For the in vivo bio-imaging experiment, $15 \mathrm{mg} / \mathrm{ml} \mathrm{D}$-luciferin potassium salt (Sigma-Aldrich) was prepared in sterile distilled water. Mice were anesthetized using isoflurane, and $200 \mu \mathrm{l}$ D-luciferin solution was peritoneally injected, followed by the quantitative bioluminescence measurement using the IVIS Lumina XRMS In Vivo Imaging System (Perkin Elmer). For the subcutaneous PC xenograft model, cells planed for the inoculation were prepared at 2.5 million cells in $50 \mu \mathrm{l}$ PBS, and mixed with $50 \mu$ l Matrigel (Corning Matrigel Matrix High Concentration) in a total of $100 \mu \mathrm{l}$ cell suspension, followed by the subcutaneous inoculation. After tumors developed to a volume of $150 \mathrm{~mm}^{3}$ (calculated by use of the modified ellipsoid formula; i.e., length $\times$ width $^{2} / 2$ ), mice were randomized into 2 groups (vehicle or VE822 treatment), with 6 mice in each group (vehicle or VE822 treatment). Mice were treated with either vehicle alone (10\% D-a-tocopherol polyethylene glycol 1000 succinate) or VE822 $(60 \mathrm{mg} / \mathrm{kg}$ in $10 \% \mathrm{D}$-a-tocopherol polyethylene glycol 1000 succinate) by oral gavage. Treatment was administered 4 times weekly for 4 weeks. Tumors were manually measured every 5 days, and mice were sacrificed when tumors reached greater than $15 \mathrm{~mm}$ in any direction.

Bioinformatic analysis. For RNA-seq, sequencing reads were aligned to the Ensembl GRCh38.88 reference genome using the RNA-specific STAR aligner (v2.3.1z4) (53). Alignment files (SAM/BAM format) were filtered to retain only primary alignments (samtools view -F 0x0100) (54). Expression was quantified for each sample at the gene level using featureCounts (v1.4.4) (55), which counts reads aligning to annotated exons (-t exon option). The raw sequence counts were normalized and tested for differential expression using Bioconductor's DESeq package (56). Heatmaps were created using GENE-E (www.broadinstitute.org/ cancer/software/GENE-E/). GO analysis was performed using DAVID bioinformatics resources (https://david.ncifcrf.gov). Clinical data sets were analyzed using Oncomine (https://www.oncomine.org/resource/ login.html), and the cBio Portal for Cancer Genomics (cBioPortal; www.cbioportal.org). The Integrative Genomics Viewer (https://www. broadinstitute.org/igv/) was used for visualization of ChIP-seq data analysis, and GSEA software was used for determining the pathways (C2 gene sets including chemical and genetic perturbations, Canonical pathways, BIOCARTA, KEGG, and REACTOME). For ChIP-seq analysis, identification of enriched regions and peak calling were performed with FDR of less than 0.05 using version 2.0 of MACS (57). ChIP peak annotation was performed using ChIPseeker (58). Identification of motifs in enriched regions of KDM5D-regulated genes was performed by the repulsive parallel MCMC algorithm (59) using default parameters. Motif validation using an annotated collection of protein-DNA-binding sequence motifs was performed using MotIV (Motif Identification and Validation, R package version 1.34.0, Mercier E and Gottardo R2014) and MotifDb (An Annotated Collection of Protein-DNA Binding Sequence Motifs, R package version 1.20.0, Shannon and Richards, 2017).

Statistics. Statistical analyses were performed using the unpaired 2-tailed Student's $t$ test, 1-way ANOVA, or 2-way ANOVA with a post hoc Tukey's honest significant difference (HSD) test when comparing, respectively, 2 or more than 2 conditions using GraphPad PRISM. $P$ values of less than 0.05 were regarded as statistically significant.

Study approval. All mouse experiments were conducted with approval from the Institutional Animal Care and Use Committee of Osaka Medical College (approval no. 29101N, Takatsuki) with adherence to Animal Research: Reporting of In Vivo Experiments (ARRIVE) guidelines by the National Centre for the Replacement Refinement \& Reduction of Animals in Research (NC3Rs).

\section{Author contributions}

KK, GSML, CJS, and PWK conceived and designed the study. TS, KT, GC, SHJ, and KH developed methodology. KK, NI, YY, KC, SYD, YZM, TI, and GSML performed experiments and acquired data. KK, TAG, SHJ, JA, GJN, CAM, HA, and LAM analyzed and interpreted data. KK, CJS, and PWK wrote the manuscript. All authors discussed the results and commented on the manuscript. HA, CJS, and PWK supervised the study.

\section{Acknowledgments}

This work was partially supported by the Department of Defense (DOD) (grant W81XWH-16-1-0639), the Foundation of Research Basis Development in Osaka Medical College, Grant-in-Aid no. 17K16821, 16K11033, and 16K11034 (Japan Society for the Promotion of Science [JSPS]), and NIH/National Cancer Institute Cancer Center Support Grant P30 CA008748.

Address correspondence to: Philip W. Kantoff, Memorial Sloan Kettering Cancer Center, 1275 York Avenue, New York, New York 10065, USA. Phone: 212.639.5851. Email: kantoff@mskcc. org. Or to: Christopher J. Sweeney, Dana-Farber Cancer Institute, 450 Brookline Ave., Boston, Massachusetts 02215, USA. Phone: 617.632.1914; Email: christopher_sweeney@dfci.harvard.edu.
1. Siegel RL, Miller KD, Jemal A. Cancer statistics, 2017. CA Cancer J Clin. 2017;67(1):7-30.

2. Komura K, Sweeney CJ, Inamoto T, Ibuki N, Azuma H, Kantoff PW. Current treatment strategies for advanced prostate cancer. Int $J$ Urol. 2018;25(3):220-231.

3. Komura K, et al. Resistance to docetaxel in prostate cancer is associated with androgen receptor activation and loss of KDM5D expression. Proc Natl Acad Sci U S A. 2016;113(22):6259-6264.

4. Lee MG, Norman J, Shilatifard A, Shiekhattar R. Physical and functional association of a trimethyl H3K4 demethylase and Ring6a/MBLR, a polycomb-like protein. Cell. 2007;128(5):877-887.
5. Chandran UR, et al. Gene expression profiles of prostate cancer reveal involvement of multiple molecular pathways in the metastatic process. BMC Cancer. 2007;7:64.

6. Grasso CS, et al. The mutational landscape of lethal castration-resistant prostate cancer. Nature. 2012;487(7406):239-243.

7. Holzbeierlein J, et al. Gene expression analysis of human prostate carcinoma during hormonal therapy identifies androgen-responsive genes and mechanisms of therapy resistance. Am J Pathol. 2004;164(1):217-227.

8. Kumar A, et al. Substantial interindividual and limited intraindividual genomic diversity among tumors from men with metastatic prostate cancer. Nat Med. 2016;22(4):369-378.

9. Taylor BS, et al. Integrative genomic profiling of human prostate cancer. Cancer Cell. 2010;18(1):11-22.

10. Tomlins SA, et al. Integrative molecular concept modeling of prostate cancer progression. Nat Genet. 2007;39(1):41-51.

11. Varambally $\mathrm{S}$, et al. Integrative genomic and proteomic analysis of prostate cancer reveals signatures of metastatic progression. Cancer Cell. 2005;8(5):393-406.

12. Yu YP, et al. Gene expression alterations in prostate cancer predicting tumor aggression and pre- 
ceding development of malignancy. JClin Oncol. 2004;22(14):2790-2799.

13. Iwase $\mathrm{S}$, et al. The $\mathrm{X}$-linked mental retardation gene SMCX/JARID1C defines a family of histone $\mathrm{H} 3$ lysine 4 demethylases. Cell. 2007;128(6):1077-1088.

14. Yamamoto S, et al. JARID1B is a luminal lineagedriving oncogene in breast cancer. Cancer Cell. 2014;25(6):762-777.

15. Subramanian A, et al. Gene set enrichment analysis: a knowledge-based approach for interpreting genome-wide expression profiles. Proc Natl Acad Sci U S A. 2005;102(43):15545-15550.

16. Di Micco R, et al. Oncogene-induced senescence is a DNA damage response triggered by DNA hyperreplication. Nature. 2006;444(7119):638-642.

17. Halazonetis TD, Gorgoulis VG, Bartek J. An oncogene-induced DNA damage model for cancer development. Science. 2008;319(5868):1352-1355.

18. Burrell RA, et al. Replication stress links structural and numerical cancer chromosomal instability. Nature. 2013;494(7438):492-496.

19. Gorgoulis VG, et al. Activation of the DNA damage checkpoint and genomic instability in human precancerous lesions. Nature. 2005;434(7035):907-913.

20. Toledo LI, et al. ATR prohibits replication catastrophe by preventing global exhaustion of RPA. Cell. 2013;155(5):1088-1103.

21. Vassin VM, Anantha RW, Sokolova E, Kanner S, Borowiec JA. Human RPA phosphorylation by ATR stimulates DNA synthesis and prevents ssDNA accumulation during DNA-replication stress. J Cell Sci. 2009;122(Pt 22):4070-4080.

22. Bhowmick R, Minocherhomji S, Hickson ID. RAD52 facilitates mitotic DNA synthesis following replication stress. Mol Cell. 2016;64(6):1117-1126.

23. Minocherhomji S, et al. Replication stress activates DNA repair synthesis in mitosis. Nature. 2015;528(7581):286-290.

24. Cimprich KA, Cortez D. ATR: an essential regulator of genome integrity. Nat Rev Mol Cell Biol. 2008;9(8):616-627.

25. Kumar A, et al. ATR mediates a checkpoint at the nuclear envelope in response to mechanical stress. Cell. 2014;158(3):633-646.

26. Peng CY, Graves PR, Thoma RS, Wu Z, Shaw AS, Piwnica-Worms H. Mitotic and G2 checkpoint control: regulation of 14-3-3 protein binding by phosphorylation of Cdc25C on serine-216. Science. 1997;277(5331):1501-1505.

27. Thanasoula M, Escandell JM, Suwaki N, Tarsounas M. ATM/ATR checkpoint activation downregulates CDC25C to prevent mitotic entry with uncapped telomeres. EMBO J. 2012;31(16):3398-3410.

28. Cottini F, et al. Synthetic lethal approaches exploiting DNA damage in aggressive myeloma. Cancer Discov. 2015;5(9):972-987.

29. Barbieri CE, et al. Exome sequencing identifies recurrent SPOP, FOXA1 and MED12 mutations in prostate cancer. Nat Genet. 2012;44(6):685-689.

30. Gaillard H, Garcia-Muse T, Aguilera A. Replication stress and cancer. Nat Rev Cancer. 2015;15(5):276-289.

31. Ozeri-Galai E, Lebofsky R, Rahat A, Bester AC, Bensimon A, Kerem B. Failure of origin activation in response to fork stalling leads to chromosomal instability at fragile sites. Mol Cell. 2011;43(1):122-131.

32. Fokas E, et al. Targeting ATR in vivo using the novel inhibitor VE-822 results in selective sensitization of pancreatic tumors to radiation. Cell Death Dis. 2012;3:e441.

33. Ross-Adams $\mathrm{H}$, et al. Integration of copy number and transcriptomics provides risk stratification in prostate cancer: A discovery and validation cohort study. EBioMedicine. 2015;2(9):1133-1144.

34. Whitington $\mathrm{T}$, et al. Gene regulatory mechanisms underpinning prostate cancer susceptibility. Nat Genet. 2016;48(4):387-397.

35. Ahearn TU, et al. A prospective investigation of PTEN loss and ERG expression in lethal prostate cancer. J Natl Cancer Inst. 2016;108(2):djv346.

36. Sinnott JA, et al. Prognostic utility of a new mRNA expression signature of Gleason score. Clin Cancer Res. 2017;23(1):81-87.

37. LaTulippe E, et al. Comprehensive gene expression analysis of prostate cancer reveals distinct transcriptional programs associated with metastatic disease. Cancer Res. 2002;62(15):4499-4506.

38. Setlur SR, et al. Estrogen-dependent signaling in a molecularly distinct subclass of aggressive prostate cancer. J Natl Cancer Inst. 2008;100(11):815-825.

39. Robinson D, et al. Integrative clinical genomics of advanced prostate cancer. Cell. 2015;161(5):1215-1228.

40. Hou J, et al. Gene expression-based classification of non-small cell lung carcinomas and survival prediction. PLoS One. 2010;5(4):e10312.

41. Sanchez-Carbayo M, Socci ND, Lozano J, Saint F, Cordon-Cardo C. Defining molecular profiles of poor outcome in patients with invasive bladder cancer using oligonucleotide microarrays. J Clin Oncol. 2006;24(5):778-789.

42. Kim SM, et al. Prognostic biomarkers for esophageal adenocarcinoma identified by analysis of tumor transcriptome. PLoS One. 2010;5(11):e15074.

43. Cerami E, et al. The cBio cancer genomics portal: an open platform for exploring multidimensional cancer genomics data. Cancer Discov.
2012;2(5):401-404.

44. Cancer Genome Atlas Research Network. The molecular taxonomy of primary prostate cancer. Cell. 2015;163(4):1011-1025.

45. Nakagawa T, et al. A tissue biomarker panel predicting systemic progression after PSA recurrence post-definitive prostate cancer therapy. PLoS One. 2008;3(5):e2318.

46. O'Hanlon LH. New tests for prostate cancer. J Natl Cancer Inst. 2013;105(22):1672-1674.

47. Cuzick J, et al. Prognostic value of an RNA expression signature derived from cell cycle proliferation genes in patients with prostate cancer: a retrospective study. Lancet Oncol. 2011;12(3):245-255.

48. Toledo LI, et al. A cell-based screen identifies ATR inhibitors with synthetic lethal properties for cancer-associated mutations. Nat Struct Mol Biol. 2011;18(6):721-727.

49. Kwok M, et al. ATR inhibition induces synthetic lethality and overcomes chemoresistance in TP53- or ATM-defective chronic lymphocytic leukemia cells. Blood. 2016;127(5):582-595.

50. Williamson CT, et al. ATR inhibitors as a synthetic lethal therapy for tumours deficient in ARID1A. Nat Commun. 2016;7:13837.

51. Wright DJ, et al. Genetic variants associated with mosaic Y chromosome loss highlight cell cycle genes and overlap with cancer susceptibility. Nat Genet. 2017;49(5):674-679.

52. Forsberg LA, et al. Mosaic loss of chromosome $\mathrm{Y}$ in peripheral blood is associated with shorter survival and higher risk of cancer. Nat Genet. 2014;46(6):624-628.

53. Dobin A, et al. STAR: ultrafast universal RNA-seq aligner. Bioinformatics. 2013;29(1):15-21.

54. Li H, et al. The Sequence Alignment/ Map format and SAMtools. Bioinformatics. 2009;25(16):2078-2079.

55. Liao Y, Smyth GK, Shi W. featureCounts: an efficient general purpose program for assigning sequence reads to genomic features. Bioinformatics. 2014;30(7):923-930.

56. Anders S, Huber W. Differential expression analysis for sequence count data. Genome Biol. 2010;11(10):R106.

57. Zhang Y, et al. Model-based analysis of ChIP-Seq (MACS). Genome Biol. 2008;9(9):R137.

58. Yu G, Wang LG, He QY. ChIPseeker: an R/Bioconductor package for ChIP peak annotation, comparison and visualization. Bioinformatics. 2015;31(14):2382-2383.

59. Ikebata H, Yoshida R. Repulsive parallel MCMC algorithm for discovering diverse motifs from large sequence sets. Bioinformatics. 2015;31(10):1561-1568. 